\title{
Therapeutic Strategies to Enhance the Anticancer Efficacy of Histone Deacetylase Inhibitors
}

\author{
Claudia P. Miller, ${ }^{1,2,3}$ Melissa M. Singh, ${ }^{1,2}$ Nilsa Rivera-Del Valle, ${ }^{1,2,4}$ \\ Christa A. Manton, ${ }^{1,2,4}$ and Joya Chandra ${ }^{1,2}$ \\ ${ }^{1}$ Department of Pediatrics Research, Children's Cancer Hospital, The University of Texas M.D. Anderson Cancer Center, \\ Houston, TX 77030, USA \\ ${ }^{2}$ The Center for Cancer Epigenetics, The University of Texas M.D. Anderson Cancer Center, Houston, TX 77030, USA \\ ${ }^{3}$ Department of Developmental Neurobiology, St. Jude Children's Research Hospital, Memphis, TN 38105, USA \\ ${ }^{4}$ Graduate School of Biomedical Sciences, The University of Texas Health Science Center at Houston, Houston, TX 77030, USA
}

Correspondence should be addressed to Joya Chandra, jchandra@mdanderson.org

Received 11 August 2010; Accepted 11 March 2011

Academic Editor: Patrick Matthias

Copyright (C) 2011 Claudia P. Miller et al. This is an open access article distributed under the Creative Commons Attribution License, which permits unrestricted use, distribution, and reproduction in any medium, provided the original work is properly cited.

\begin{abstract}
Histone acetylation is a posttranslational modification that plays a role in regulating gene expression. More recently, other nonhistone proteins have been identified to be acetylated which can regulate their function, stability, localization, or interaction with other molecules. Modulating acetylation with histone deacetylase inhibitors (HDACi) has been validated to have anticancer effects in preclinical and clinical cancer models. This has led to development and approval of the first HDACi, vorinostat, for the treatment of cutaneous $\mathrm{T}$ cell lymphoma. However, to date, targeting acetylation with HDACi as a monotherapy has shown modest activity against other cancers. To improve their efficacy, HDACi have been paired with other antitumor agents. Here, we discuss several combination therapies, highlighting various epigenetic drugs, ROS-generating agents, proteasome inhibitors, and DNA-damaging compounds that together may provide a therapeutic advantage over single-agent strategies.
\end{abstract}

\section{Introduction}

Over time, an appreciation of the importance and complexity of epigenetic events, such as DNA methylation, histone posttranslational modifications, and miRNA regulation, has fueled interest in many new areas of research. Histone acetylation is one process that is being intensely studied due to its ability to regulate gene transcription. The enzymes that regulate histone acetylation are often inappropriately expressed in cancer cells, which can lead to the silencing of tumor suppressor genes or activation of oncogenes. Because of this, many of these enzymes have become popular targets for cancer therapy. In this paper we will highlight histone deacetylase inhibitors, a group of compounds that blocks the zinc-dependent histone deacetylases involved in removing acetyl groups from lysine residues. Modulation of protein acetylation by the first-in-class FDA (U S Food and Drug Administration) approved HDACi, vorinostat, has been shown to be successful for the treatment of refractory cutaneous $\mathrm{T}$ cell lymphoma (CTCL). However, despite the promising results employing HDACi as an epigenetic targeted therapy, its limited success in specific cancers as a single drug has prompted further investigation of combining HDACi with other anticancer agents. These combination regimens, which will be the focus of this review, may enhance the clinical efficacy of HDACi and may provide a therapeutic advantage in cancers where HDACi alone have limited activity.

\section{Histone Deacetylases (HDACs) and Cancer}

Histone deacetylases (HDACs) are a group of enzymes that, in conjunction with histone acetyltransferases (HATs), regulate the acetylation status of histone tails. HATs acetylate lysine residues on histone tails resulting in neutralization of their charge and decreased affinity for DNA [1]. This 
"loosening" of the histone-DNA interaction is associated with conformational changes which allow for transcription factors to bind to the DNA and impact gene transcription [2]. HDACs, on the other hand, remove acetyl groups which lead to a more compact chromatin conformation that is often associated with gene repression. Importantly, HDACs usually do not function alone, but are part of multiprotein complexes that contain DNA-binding proteins, chromatinremodeling proteins, and other histone-modifying proteins that participate together to regulate transcription. In addition, according to the "histone code" hypothesis, histone modifications work together with other epigenetic modifications to determine certain transcriptional outcomes [3].

HDACs are categorized into four families, class I, II, III, and IV, based on their structure. Class I, which includes HDAC $1,2,3$, and 8 , is predominately localized to the nucleus. Class II consists of HDACs 4, 5, 6, 7, 9, and 10 and is detected in both the nucleus and cytoplasm. HDAC 11 is the sole class IV member and resides in the nucleus [4]. These three classes of HDACs are zinc-dependent enzymes and are the molecular targets of HDACi. In contrast, class III is comprised of the NAD-dependent deacetylases, sirtuins (SIRT 1-7), which are found in the nucleus, cytoplasm, and mitochondria and have been identified to be involved in metabolism and aging [5]. However, they will not be discussed in this paper since they are not targets of HDACi.

There are numerous studies demonstrating that histones are not the only substrates for HDACs and HATs. These enzymes also regulate acetylation of nonhistone proteins, including transcription factors, chaperone proteins, and signaling molecules involved in cancer development and progression such as the tumor suppressor p53 [6]. In general, acetylation can interfere with binding, function, and/or stability (turnover) of the protein. Since HDACs are involved in deacetylating a wide variety of substrates they have been identified to modulate many cellular processes and thus may be used by cancer cells for a survival advantage. Based on this rationale, efforts to define which HDACs are involved in cancer development and progression are being undertaken. Many of these studies have employed HDACi to demonstrate the validity of HDACs as therapeutic targets, but effects were selective to cancer type or were inhibitor specific. However, strategies using small interfering RNA (siRNA) against class I and II HDACs have been used to determine which HDACs play a role in proliferation and survival of cancer cells. Silencing of HDAC 1 and 3 by siRNA resulted in antiproliferative effects in human cervical carcinoma cells (HeLa) [7]. However, silencing class II HDACs, HDAC 4 and 7, did not have an effect on proliferation [7]. Additionally, HDAC 3 knockdown by siRNA resulted in hyperacetylation of histone-H3 and an increase in apoptotic cell death [7]. These results suggest that, at least in the case of cervical carcinoma, class I HDACs may be better candidates for inhibition over class II isoforms. Yet, it is difficult to pinpoint which HDACs are suitable targets since examination of HDAC expression levels differs greatly from cancer to cancer, with many displaying aberrant levels. In some cases, even the surrounding normal tissue may also express high levels of HDACs, begging the question of how only malignant cells can be targeted with HDACi and not healthy cells. Surprisingly, studies show that HDACi selectively target tumor cells at doses that have very little effect on normal cells [8-10]. This susceptibility of transformed cells to HDACi therapy is probably due to their dependence on HDACs for modulating expression of genes involved in tumor cell growth, differentiation, and apoptosis that provide cancer cells with a survival advantage (further discussed Section 4) [11]. Because of this reliance, interfering with HDACs for a therapeutic advantage in cancer is gaining momentum.

\section{Development of Histone Deacetylase Inhibitors (HDACi) for Cancer Therapy}

Initially, HDACi were identified by several groups as agents that induced differentiation of murine erythroleukemia cells (MELC). Transfection experiments in MELC performed by Friend et al. revealed that treatment with dimethyl sulfoxide (DMSO) resulted in synthesis and accumulation of hemoglobin, an indication of erythroid differentiation [12]. Similar results were also observed by Yoshida et al. in experiments that demonstrated differentiation of MELC with a naturally derived antifungal antibiotic, trichostatin A (TSA) [13]. Further analysis of TSA in rat fibroblasts showed that this compound induced G1 and G2 cell cycle arrest but, most importantly, subsequent studies analyzing histone modifications identified histone deacetylases as the molecular targets for TSA. During this time, Paul Marks' group discovered that HMBA, a small molecule polar compound, was able to induce differentiation similarly to DMSO [14]. Compounds that share certain structural features with DMSO have now been synthesized in an attempt to generate compounds with increased anticancer efficacy [15]. One of these compounds is suberoylanilide hydroxamic acid (SAHA), now known as vorinostat. However, the targets of these differentiating compounds were still unknown until closer examination of the structure of vorinostat revealed a similarity to the structure of TSA. Based on these observations, Marks' group reported in 1998 that vorinostat targeted HDACs, inhibiting HDAC 1 and 3 resulting in acetylation of histones in various cancer cell lines [15]. Subsequent studies over the next eight years demonstrated that vorinostat modulated transcription of gene expression and had antitumor selectivity in in vivo cancer models. Eventually, this led to its evaluation in clinical trials and the FDA approval of the first-in-class HDACi for cancer treatment.

Since these events, several HDACi have been identified either through synthetic or natural sources. HDACi can be separated into several structurally distinct classes: shortchain fatty acids (i.e., valproic acid), hydroxamic acids (i.e., vorinostat, TSA, and PCI-24781), benzamides (i.e., entinostat), cyclic tetrapeptides (i.e., depsipeptide), and electrophilic ketones. Some of these compounds selectively block specific classes of HDACs, while some have a broader spectrum of activity and therefore inhibit several classes of HDACs. For example, the benzamide entinostat (previously known as SNDX-275 or MS-275) is selective for class I HDACs (HDAC 1,2 , and 3 ), while the HDACi tubacin specifically targets 
only HDAC 6 [16]. In contrast, the majority of hydroxamic acids, including vorinostat, panobinostat (LBH589), and TSA, behave as pan-HDACi, blocking several classes of HDACs, although more selective hydroxamic acid inhibitors are being developed. For example, a novel hydroxamic acid, PCI-24781, is currently in phase I clinical trials, preferentially inhibits class I and II HDACs, and is more potent at targeting these isoforms compared to vorinostat [17].

\section{HDACi: Mechanisms of Action}

Treatment with HDACi has been found to change the gene expression of about $7 \%$ of the genes studied, indicating that HDACi can be used to alter a subset of genes [18]. In fact, gene expression analyses have demonstrated that HDACi can selectively induce apoptosis in cancer cells by upregulating and/or downregulating the expression of proapoptotic and antiapoptotic genes [19]. Mechanistic studies have implicated activation of the death-receptor (extrinsic) pathway or the mitochondrial (intrinsic) apoptotic pathways as a mechanism of action of different HDACi. Induction of distinct cell death pathways may be associated with the structurally diverse HDACi, which have different targets and have been demonstrated to be cell-type dependent.

The role of HDACi in triggering the extrinsic apoptotic pathway has been demonstrated by several in vitro studies. In these experiments, HDACi have shown to activate death receptors, including TRAIL, DR5, FAS, and TNF alpha [19]. These observations have been validated by studies where the inhibition of death receptors and their ligands abrogated HDACi-dependent apoptosis $[10,20]$. In addition, in vivo experiments suppressing TRAIL and Fas by siRNA in mice resulted in a significant reduction in apoptosis after treatment with the HDACi, valproic acid [21]. Yet, several studies have also implicated the involvement of the intrinsic pathway in HDACi-induced apoptosis. HDACi can transcriptionally regulate the expression of proapoptotic $\mathrm{BH} 3$-only proteins including Bid, Bad, and Bim, which play an important role in the activation of the intrinsic apoptotic pathway [22-24]. Furthermore, elevated levels of reactive oxygen species (ROS) have been observed after treatment with HDACi [24-26]. The increase in ROS has been shown to precede changes in mitochondrial membrane potential [27], suggesting a link between HDACi, ROS, BH3-only proteins and activation of the intrinsic pathway.

In fact, oxidative stress has been identified as a mechanism by which HDACi may be exerting its lethal effects in tumor cells. However, the manner by which HDACi induce oxidative stress is not well understood. Two prominent mechanisms have been reported. One involves mitochondrial injury, while the other implicates modulating antioxidants levels (Figure 1). In an acute leukemia treated cell line, vorinostat induced apoptosis by the expression of proapoptotic Bid, which resulted in disruption of mitochondria, a major source of ROS in the cell. Accordingly, subsequent production of ROS was observed in these cells (Figure 1(a)) [24]. More recently, detailed studies by Paul Marks' laboratory have demonstrated that vorinostat and entinostat upreg- ulate thioredoxin binding protein-2 (TBP-2), which is a protein that binds and inhibits thioredoxin (Trx) (Figure 1(b)) [28]. Trx is a ubiquitous protein with pleiotropic effects, with one of its major functions to operate as an intracellular antioxidant. Interestingly, studies have shown that this antioxidant is upregulated in certain types of tumors $[29,30]$ perhaps giving cancer cells a survival advantage to deal with the elevated oxidative stress. These findings indicate that Trx may be a good candidate to target for the treatment of cancer. Ungerstedt and colleagues demonstrated that exposure of transformed cells to HDACi resulted in ROS-dependent apoptosis. Furthermore, nontransformed cells were resistant to this HDACi treatment; instead an increase in Trx levels was detected, and no production of ROS was observed. The rise of HDACi-induced Trx expression in nonmalignant cells offered cytoprotection since siRNA against the antioxidant resulted in increased oxidative stress and sensitivity towards HDACi [9]. These observations of Trx overexpression offering a protective mechanism against HDACi provides an additional explanation of the selectivity of HDACi for some cancer cells compared to nontransformed cells.

HDACi have also been shown to induce cell cycle arrest. The mechanism by which HDACi induce cell cycle arrest includes the induction of cell cycle genes like CDKN1A which encode the production of $\mathrm{p} 21^{\mathrm{WAF1} / \mathrm{CIP} 1}[22,31]$. Also, HDACi can transcriptionally repress cyclin D and cyclin A genes resulting in the loss of CDK2 and CDK4 kinase activity $[32,33]$. In addition to the induction of apoptosis and cell cycle arrest, HDACi have been shown to have antiangiogenic effects by downregulating proangiogenic genes like vascular endothelial growth factor (VEGF) and endothelial nitric oxide synthase (eNOS) [34]. These antiangiogenic effects have been observed in different cancer models both in vitro and in vivo [34-36]. These studies support the possibility of HDACi interfering with the metastatic process. However, more studies are needed to understand better their role in metastasis.

\section{HDACi in the Clinic}

Preclinical studies of HDACi, in cell lines and animal models, have proven to be very successful as single-modality agents for the treatment of a variety of cancers. As a result, several structurally different HDACi have been used in hundreds of clinical trials to test their toxicity and efficacy. In general, clinical trials involving HDACi alone, or in combination with other chemotherapeutic agents, yield promising results and demonstrate biological and antitumor activity.

Vorinostat is the first HDACi to show promise in the clinic. In phase I and II trials, vorinostat was well tolerated and $\sim 30 \%$ of CTCL patients enrolled in the study received clinical benefit [37]. However, in other phase II trials evaluating the efficacy of vorinostat in solid tumors, including ovarian [38], breast, colorectal, nonsmall cell lung [39], head and neck [40], and glioblastoma [41], only a moderate effect was observed. Moreover, treatment of metastatic tumors with vorinostat had limited success [40, 42, 43]. 


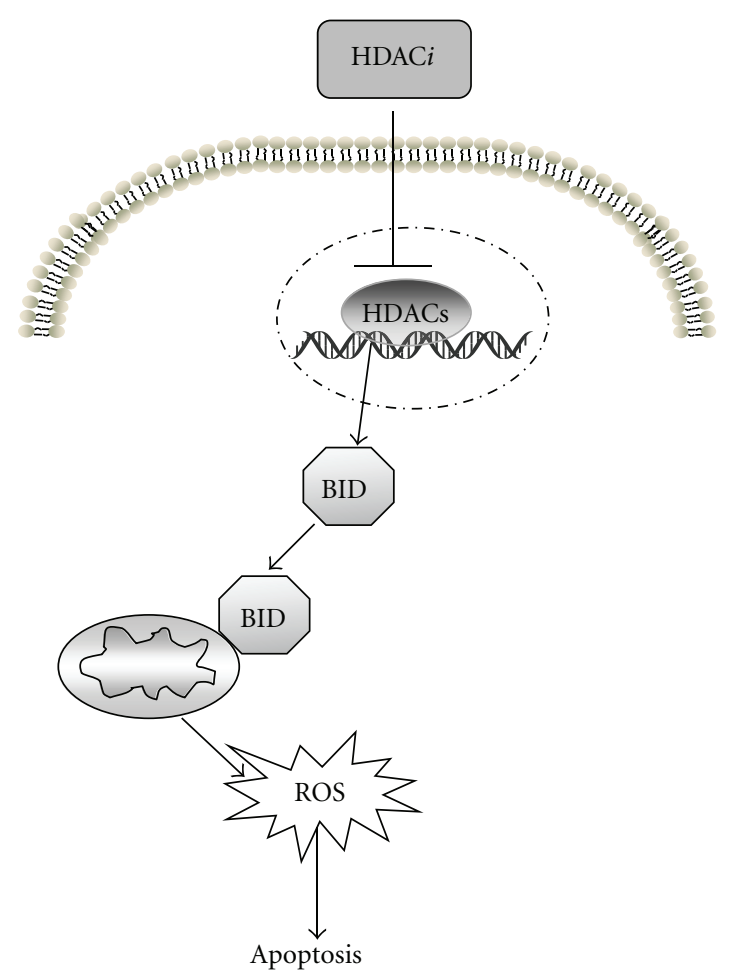

(a)

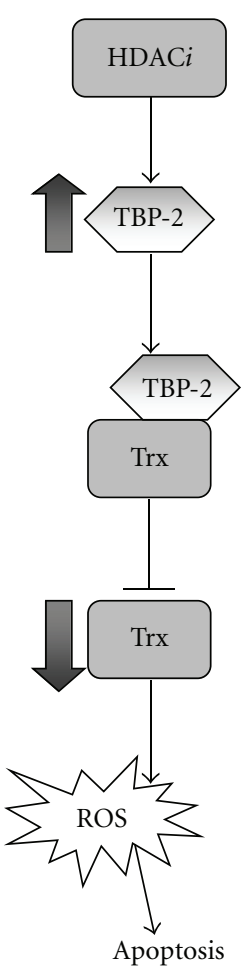

(b)

FIGURe 1: Proposed mechanisms by which HDACi induce ROS. (a) Mitochondrial injury. HDACi induces expression of Bid protein. This proapoptotic molecule binds to and disrupts the mitochondrial membrane, which results in increased ROS levels and apoptosis. (b) Alterations in antioxidant levels. HDACi upregulate the expression of thioredoxin-binding protein-2 (TBP-2). TBP-2 binds to and inhibits the antioxidant thioredoxin (Trx). This inhibition results in an imbalance of oxidants and antioxidants leading to increased ROS levels, which promotes apoptosis.

Upon the success of vorinostat in CTCL and its approval by the FDA for this disease, several new HDACi were developed and have been investigated in clinical trials (reviewed in [44]). There are currently close to 50 active clinical trials evaluating several HDACi as monotherapies for a variety of cancers and over 100 trials combining HDACi with other chemotherapeutic agents (http://www.clinicaltrials.gov/, http:// www.cancer.gov/clinicaltrials). These studies include panHDACi, such as the novel compound PCI-24781 (Pharmacyclics), as well as isoform-specific HDACi, such as entinostat. Preclinical studies using PCI-24781 demonstrate an inhibition in cell growth and an increase in apoptosis, and treatment of colon tumor xenografts significantly reduces tumor volume [17]. PCI-24781 is currently under evaluation in phase I trials for sarcoma, non-Hodgkin lymphoma, multiple myeloma, and chronic lymphocytic leukemia. Another structurally different $\mathrm{HDACi}$, entinostat, is more selective for class I HDACs. Like vorinostat, entinostat shows the greatest therapeutic response in patients with leukemia and lymphoma $[45,46]$ whereas it is only moderately effective for solid tumors $[47,48]$. Entinostat is currently being evaluated as mono- and combination therapies for a variety of cancer types (Table 1).

Despite promising preclinical in vitro and in vivo data evaluating $\mathrm{HDACi}$, clinical trials using these agents as monotherapies have mostly been successful in treating CTCL and hematological malignancies. One explanation for this observation is the inability to achieve appropriate doses of $\mathrm{HDACi}$ and consistent acetylation of target proteins. In vitro studies evaluating HDACi require at least 24 hours and micromolar concentrations to cause tumor cell death. Phase I clinical trials of vorinostat demonstrate that plasma concentrations $\left(C_{\max }\right)$ reach the micromolar range, $2.5 \mu \mathrm{M}$ for oral administration of $400 \mathrm{mg} / \mathrm{d}$ and $9 \mu \mathrm{M}$ for $300 \mathrm{mg} / \mathrm{m}^{2}$ per day for intravenous administration [49]; however the half life is relatively short, 91.6-127 minutes orally and 34.742.4 minutes intravenously [50]. These data suggest that continuous administration of these agents may be necessary to achieve clinical response. This may not be feasible with a broad-spectrum HDACi, like vorinostat, due to the large number of acetylated target proteins which may contribute to dose-limiting toxicities. HDACi clinical trials primarily focus on evaluating the acetylation status of histone $\mathrm{H} 3$ and $\mathrm{H} 4$ to determine whether these compounds are blocking their substrates. These pharmacodynamic studies have revealed that increased acetylation of histone $\mathrm{H} 3$ and $\mathrm{H} 4$ is observed in peripheral blood mononuclear cells (PMBCs) and/or bone marrow mononuclear cells (BMMCs) of patients treated with HDACi, indicating that these inhibitors are targeting HDACs. However, similar increased acetylation has been detected in both responders and nonresponders suggesting that increased histone acetylation in PMBCs and BMMCs 
TABLE 1: HDACi combination regimens currently in clinical trials. Partial list of clinical trials evaluating the combination of HDACi with other anticancer agents discussed in this paper (source: http://www.clinicaltrials.gov/, http://www.cancer.gov/clincialtrials).

\begin{tabular}{|c|c|c|}
\hline HDACi & Other Intervention & Tumor Type \\
\hline Entinostat (SNDX-275) & azacitidine & leukemia, MDS, colorectal cancer, NSCLC \\
\hline Mocetinostat (MGCD0103) & azacitidine & MDS \\
\hline \multirow{6}{*}{ Panobinostat (LBH589) } & azacitidine & MDS, CML, AML \\
\hline & bortezomib & $\begin{array}{l}\text { peripheral T cell lymphoma, NK/T cell } \\
\text { lymphoma, MM, pancreatic cancer }\end{array}$ \\
\hline & bortezomib, dexamethasone & $\mathrm{MM}$ \\
\hline & decitabine & MDS, AML \\
\hline & decitabine, temozolomide & melanoma \\
\hline & radiation therapy & $\begin{array}{l}\text { prostate cancer, esophageal cancer, head and neck } \\
\text { cancer }\end{array}$ \\
\hline PCI-24781 & doxorubicin & sarcoma \\
\hline Romidepsin & bortezomib & myeloma \\
\hline \multirow{5}{*}{ Valproic acid } & azacitidine & advanced cancers \\
\hline & azacitidine, ATRA & AML, MDS \\
\hline & decitabine, ATRA & AML \\
\hline & radiation therapy, bevacizumab & children with high grade gliomas \\
\hline & radiation therapy, temozolomide & high grade gliomas, brain tumors \\
\hline \multirow{10}{*}{ Vorinostat } & azacitidine & $\begin{array}{l}\text { AML, MDS, DLBCL, lymphoma, MM, NSCLC, } \\
\text { Non-Hodgkin's lymphoma }\end{array}$ \\
\hline & bortezomib, AMG 655 & lymphoma \\
\hline & bortezomib, dexamethasone & $\mathrm{MM}$ \\
\hline & $\begin{array}{l}\text { cisplatin, pemetrexed, radiation } \\
\text { therapy }\end{array}$ & NSCLC \\
\hline & cisplatin, radiation therapy & squamous cell carcinoma \\
\hline & gemtuzumab ozogamicin, azacitidine & AML \\
\hline & marizomib (NPI-0052) & NSCLC, pancreatic cancer, melanoma, lymphoma \\
\hline & paclitaxel, radiation therapy & NSCLC \\
\hline & radiation therapy & $\begin{array}{l}\text { brain metastases, NSCLC, pancreatic cancer, } \\
\text { pelvic cancer }\end{array}$ \\
\hline & radiation therapy, fluorouracil & pancreatic adenocarcinoma \\
\hline
\end{tabular}

ATRA: all-trans retinoic acid, MM: multiple myeloma, NSCLC: nonsmall cell lung carcinoma, AML: acute myeloid leukemia, CML: chronic myelogenous leukemia, MDS: myelodysplastic syndrome, and DLBCL: Diffuse large B-cell lymphoma.

does not correlate with clinical response. Histones are not the only proteins capable of being acetylated. Our knowledge of acetylated nonhistone proteins is rapidly increasing through efforts to define the acetylome [51] but the biological relevance of acetylation on many of these proteins is still mostly unknown. A greater understanding of the acetylome may reveal other molecular endpoints that might indicate a favorable clinical response or that might identify contributors of dose-limiting toxicities. In addition, deciphering the individual role of each individual HDAC in cancer progression will aid in knowing which specific isoform to target. However, even isoform-specific inhibitors may not completely eliminate on-target dose-limiting toxicities because most HDACs reside in multiple large multiprotein complexes. A single HDAC can simultaneously play different roles within the cell depending on which complex it is associated. In addition to the specificity of HDACi, the lack of response of some patients to HDACi therapy may be attributed to mechanisms of resistance [50].

Overall, HDACi have shown promise in the clinic but there is clearly room for improvement of therapeutic index. One way to achieve greater clinical efficacy is to use HDACi in combination with other chemotherapeutic agents. In fact, many preclinical studies provide evidence supporting synergistic or additive effects of HDACi in combination with other cytotoxic agents, and a partial list of HDACi combination trials is found in Table 1 . The agents listed will be the focus of the remainder of the paper.

\section{Combining HDACi with Other Epigenetic Therapies}

The predominant function of HDACs is the modification of histone tails which influences gene transcription. In addition 
to histone acetylation, gene transcription is also controlled by DNA methylation and histone methylation. These processes often work in concert with one another providing the rationale for combining epigenetic therapies for cancer treatment.

6.1. DNA Methyltransferases. DNA methylation involves the covalent addition of a methyl group to cytosine residues of DNA by enzymes termed DNA methyltransferases (DNMTs). Often, DNA methylation occurs within CpG islands located within the $5^{\prime}$ promoter regions of genes [52]. DNA methylation can inhibit transcription directly by influencing transcription factor binding [53] but also maintains chromatin in a transcriptionally inactive state through the recruitment of methyl-CpG binding proteins (MBDs) [54, 55], some of which recruit histone deacetylases for added epigenetic control $[56,57]$. In normal cells, the CpG islands of transcriptionally active genes are not methylated [52]. However, in cancer, many of the unmethylated genes become aberrantly methylated [58-60]. The discovery that CpG methylation was a causative event in tumor progression led to the search for drugs which could reverse the DNA methylation and restore gene expression.

The first-FDA approved DNMT inhibitors (DNMTi) are the nucleoside analogs 5-azacytidine (azacitidine) and 5-aza2 '-deoxycytidine (decitabine). Nucleoside analogs, as well as nonnucleoside analog DNMTi, are effective anticancer agents that cause increased apoptosis [61]. Moreover, these agents reverse the DNA hypermethylation association with certain cancer genes and alter gene expression [61-63]. However, there is a wide variation among the different agents which led to the hypothesis that DNA methylation influences the stability of other chromatin marks prompting the evaluation of combined use of DNMTi and HDACi [64].

Several preclinical studies evaluating the effects of DNMTi in combination with HDACi demonstrate synergistic anticancer activity. For example, cotreatment of prostate cancer cells [65] or pancreatic cancer cells [66] with decitabine and TSA led to reduced cell proliferation which was accompanied by increased apoptosis. Similar results were obtained in other cell lines where enhanced apoptosis was observed in AML1/ETO-positive acute myelogenous leukemia (AML) cells [67] and decreased cell proliferation was observed in lung cancer cells [68] treated with depsipeptide and decitabine. Moreover, entinostat and azacitidine display synergistic cytotoxicity and apoptosis in leukemia cells which correlates with enhanced rates of histone acetylation as well as elevated intracellular reactive oxygen species [26]. Given the promising preclinical data combining HDACi with DNMTi, several clinical trials utilizing the combination were administered to patients with hematologic and solid tumors where many of the patients had minimal side effects and some achieved complete and partial remissions [69, 70]. Currently, there are 14 trials evaluating DNMTi with HDACi (Table 1).

6.2. Histone Demethylases. The methylation status of histones also plays an important role in gene expression. Although, for many years, histone methylation was considered to be a stable, irreversible modification, recently, two families of enzymes have been discovered which function to remove methyl groups from the lysines of histone and nonhistone proteins. The first enzyme to be discovered was the lysinespecific demethylase 1 (LSD1) which functions similar to the amine oxidase family of enzymes [71]. The second family of enzymes discovered are the jumonji-domain-containing proteins [72]. These metalloenzymes mediate hydroxylationbased demethylation of lysines [72]. Both of these families of demethylase enzymes have been reported to reside in complexes containing HDACs $[73,74]$, and the activity of LSD1 is influenced by HDAC function [74], providing the rationale for targeting both enzymes as epigenetic therapy.

Due to the structural similarity between LSD1 and the amine oxidase family of enzymes, several groups have demonstrated that mono- and polyamine oxidase inhibitors also target LSD1 [74-79]. Studies from our laboratory evaluating cotreatment of glioblastoma cells with the combination of HDACi, vorinostat or PCI-24781, with the LSD1 inhibitor, tranylcypromine, show a synergistic increase in apoptotic cell death [80]. Moreover, treatment of normal human astrocytes with the same doses of HDACi and tranylcypromine did not yield enhanced cell death suggesting that the synergistic apoptosis induced by the combination is selective for glioblastoma cells [80]. These data support the use of HDACi and LSD1 as combination therapy in preclinical mouse studies. In addition, future studies aimed at understanding the molecular mechanisms by which HDACs and LSD1 regulate cancer cell growth have the potential to identify new molecular targets for therapy.

Not only there is cross-talk between LSD1 and HDACs, but also LSD1 is required for the maintenance of global DNA methylation [81]. Moreover, LSD2, a homolog of LSD1, participates in establishing maternal genomic imprints during oogenesis [82]. These data suggest that targeting the LSD family and DNMTs may enhance the antitumor activity of these drugs. In fact, inhibition of LSD1 in human colorectal cells with a combination of novel oligoamine analogs and DNMTi led to a greater re-expression of aberrantly silenced genes when compared to either agent used alone [83]. In addition, colorectal xenograft models treated with the combination of PG-11144, an oligoamine analog inhibitor, and azacitidine caused dramatic decreases in tumor cell growth demonstrating the therapeutic efficacy of this combination [83].

The discovery of lysine demethylases and the influence these enzymes have on many biological processes has led to the recognition of their potential as a therapeutic target in a variety of diseases, including cancer. Developing more specific inhibitors for the demethylase enzymes, particularly the jumonji-domain-containing family where investigators are just now starting to identify inhibitors [84], will aid in understanding what role individual demethylases play during cell growth and development. These studies will make substantial contributions to our knowledge regarding epigenetic regulators and are needed to utilize epigenetic therapies to their fullest potential. 


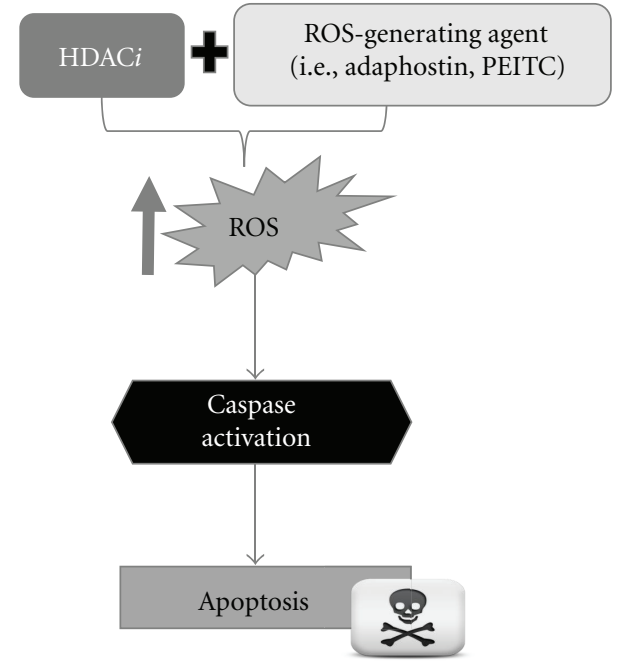

FIgURE 2: Activation of apoptosis by combinations of HDACi and ROS-generating agents. HDACi combined with agents like adaphostin and PEITC result in an increase production of ROS in cancer cells. The high levels of ROS result in induction of apoptosis via caspase activation.

\section{HDACi and ROS-Generating Agents}

Several reports have shown that HDACi induce oxidative stress in different types of cancer cells [85]. Cancer cells also have higher levels of reactive oxygen species (ROS) compared to normal cells, most likely as a consequence of an active metabolism and more robust proliferation rates [86, 87]. This difference has been used as a therapeutic strategy to treat cancer. Combining HDACi with agents that cause further oxidative stress might enhance the efficacy of HDACi for the treatment of cancer (Figure 2).

7.1. Adaphostin. Adaphostin is a drug that is part of the tyrphostin family of tyrosine kinase inhibitors, and it affects a number of different kinases. It is an analog of AG957, a drug that was originally developed to inhibit p $210^{\mathrm{Bcr} / \mathrm{abl}}$ [88]. However, studies have demonstrated that adaphostin's activity is not restricted by the presence or absence of $\mathrm{Bcr} / \mathrm{Abl}$ kinase [89]. This compound has been identified as a potential anticancer agent to treat acute leukemias such as AML and ALL (acute lymphoblastic leukemia). Importantly, adaphostin demonstrates selectivity for leukemia cells as compared to normal lymphocytes [85]. Mechanistic studies have demonstrated that adaphostin elevates levels of intracellular ROS, resulting in apoptosis [90]. Additional investigations by Le et al. expand these observations by showing that the increase in ROS in cells treated with adaphostin is the result of its accumulation in the mitochondria, where adaphostin binds to complex III, inhibiting electron transport [91] and leading to oxidative stress. Moreover, transcriptional and proteomic analyses of adaphostin-treated cells demonstrated an upregulation of oxidative stress-related genes and antioxidants [92], including the genes encoding heat shock proteins, glutathione S-transferase (GST), and superoxide dismutase. A decrease in the antioxidant glutathione has also been observed in studies in CML (chronic myelogenous leukemia) cells treated with adaphostin [90]. Taken together, these observations indicate that adaphostin is a redox-modulatory agent and a good candidate to combine with HDACi.

Unpublished data from our group shows strong synergy between two structurally different HDACi (entinostat and vorinostat) and adaphostin resulting in apoptosis in leukemia cells. Results showed a threefold increase in DNA fragmentation, a hallmark of apoptosis, when cells were treated with adaphostin combined with entinostat compared to cells treated with HDACi alone. A more potent effect was achieved with adaphostin and vorinostat, demonstrating a sixfold increase in DNA fragmentation. Furthermore, these combinations enhanced superoxide levels, suggesting that oxidative stress plays a role in the synergistic induction of apoptosis. The results observed with these combinations lend support to the idea of enhancing the efficacy of HDACi by modulating ROS levels with an oxidant-generating agent that may push the balance towards oxidative stress and cell death and provide a therapeutic advantage for the treatment of cancers such as leukemia.

7.2. $\beta$-Phenylethyl Isothiocyanate (PEITC). A second redoxmodulatory agent, which has shown promises for the treatment and prevention of cancer, is PEITC. This agent is a natural compound found in cruciferous vegetables like cauliflower, broccoli, and cabbage. PEITC has been shown to be effective in cancer cells by inhibiting carcinogenesis and inducing cell growth arrest and apoptosis [93]. Studies in prostate cancer cells demonstrate that PEITC induce apoptosis by decreasing the levels of the antiapoptotic proteins $\mathrm{Bcl}-2$ and $\mathrm{Bcl}-\mathrm{X}_{\mathrm{L}}$ [94]. In addition, it has been shown to downregulate and facilitate the degradation of the androgen receptor [95]. More detailed mechanistic studies have revealed the main mechanism by which PEITC works as an anticancer agent is through redox-modulating mechanisms. These mechanisms include the inhibition of cytochrome P450 and the induction of metabolizing enzymes like $\mathrm{NAD}(\mathrm{P}) \mathrm{H}$ : quinone oxidoreductase (NQO-1) and GST [93]. A more recent study reports production of ROS by PEITC to be mediated by inhibition of complex III and oxidative phosphorylation [96]. In addition, in vitro and in vivo studies have demonstrated PEITC to induce an accumulation in ROS that is mediated by the depletion of the antioxidant, glutathione (GSH) [97]. Furthermore, the increase of ROS and depletion of GSH by this agent have been shown to overcome the resistance of leukemia cells to fludarabine [98].

Taking into consideration that PEITC is a ROS modulating agent, it can perhaps be combined with HDACi to improve its efficacy. Recently, $\mathrm{Hu}$ et al. addressed this assumption and demonstrated that depletion of GSH by PEITC increases sensitivity to vorinostat in leukemia cells. Furthermore, the combination of HDACi/PEITC also induced ROS accumulation and apoptosis in a vorinostat-resistant cell line via activation of the NADPH oxidase. Induction of ROS by this combination allows for the translocation of transcription 
factor Nrf2 to the nucleus, stimulating transcription of genes involved in the glutathione system [99].

All together, these studies suggest that the regulation of oxidative stress plays an important role in the cytotoxic effects with HDACi and ROS generating agents. Further understanding how HDACi, alone or in combination with other redox-modulating agents, regulate oxidative stress will help in the development of better therapeutic strategies for clinical utility. Presently there are multiple clinical trials combining HDACi with other chemotherapies that together have been demonstrated to increase ROS. Some of these agents include proteasome inhibitors and DNA-damaging agents. The combination of HDACi with these agents will be discussed in the next two sections.

\section{Proteasome Inhibitors and HDACi Regimens}

A growing body of work in the literature is providing evidence to support the use of proteasome inhibitors as an option to combine with HDACi. The molecular targets of these compounds are the enzymatic activities housed within the proteolytic chamber of the proteasome. These include the chymotrypsin-, caspase-, and trypsin-like proteolytic activities that are responsible for degrading the majority of intracellular proteins [100]. Thus, inhibiting the proteasome will influence many signaling pathways and cellular processes, including cell growth and survival, tumor suppression, and apoptosis. Surprisingly, most likely due to their rapid protein turnover rate, cancer cells rely heavily on the proteasome to dispose of unwanted proteins and therefore are more susceptible to proteasome inhibition compared to nontransformed cells $[101,102]$. One of the most commonly reported consequences of proteasome inhibition in tumor cells is cell death. As a result of this selectivity, similar to HDACi, compounds that target the proteasome have emerged as novel cancer therapies in the recent years. However, despite the promise of both HDACi and proteasome inhibitors in preclinical and in vivo models as single agents, similar responses have not been duplicated in clinical settings. One way to overcome these unexpected shortcomings has been to combine these two different compounds to enhance their antitumor activity. Specifically, three clinically relevant proteasome inhibitors-bortezomib, marizomib (formerly known as NPI-0052), and carfilzomib-are proving to be strong candidates for combination regimens with HDACi.

8.1. Bortezomib. Originally synthesized as an inhibitor of the chymotrypsin-like activity of the proteasome, the boronic acid-derived compound bortezomib is the sole FDA-approved drug of its class for multiple myeloma (MM) and mantle cell lymphoma (MCL). This reversible inhibitor has been shown to work in combination with HDACi by inducing cytotoxic effects mediated primarily by cellular stress, JNK (Jun $\mathrm{NH}_{2}$-terminal kinase) activation, and upregulation of proapoptotic proteins. Stress to the cell is a commonly characterized event produced by the combination treatment that triggers apoptosis in cancer cells, and two major sources of stress stand out (Figure 3). The first one involves the generation of reactive oxygen species. Pretreatment with bortezomib sensitized MM cells to two different HDACi, vorinostat and sodium butyrate, inducing synergistic apoptosis [103]. Mitochondrial injury, JNK activation, caspase activation, and increased oxidative stress were among the events observed with the combination regimen. Importantly, treatment with an antioxidant markedly decreased JNK activation and apoptosis suggesting that ROS was contributing to these effects [103]. Similar ROS-dependent apoptosis was also observed in $\mathrm{Bcr} / \mathrm{Abl}+$ leukemic cells with the same combination treatment. Moreover, cell death was induced by bortezomib/HDACi in Gleevec-resistant K562 (Bcr/Abl+ CML) cells and patient-derived CD134+ cells that were refractory to Gleevec therapy [104]. These findings indicate that this proteasome inhibitor/HDACi regimen may provide benefits in cancers that have acquired resistance to their current therapies. Cytotoxic oxidative stress and DNA damage have also been reported in MM cells when bortezomib was paired with another HDACi, PXD101 [105]. Bhalla et al. report greater lethality in lymphoma cells when bortezomib was combined with PCI-24781 compared to single-agent treatment. The cell death observed in this model system was also ROS dependent [106]. Furthermore, gene expression analyses revealed downregulation of antioxidant genes with PCI-24781, and these effects were further enhanced when combined with bortezomib [106]. These results hint at a process by which oxidative stress may be altered when pairing HDACi with bortezomib. However, the source of ROS when these molecular-targeted therapies are combined is not completely clear. For proteasome inhibitors as single agents, studies in nonsmall cell lung cancer using peptide inhibitors seem to suggest that the mitochondrial electron transport chain is involved in producing oxidative stress [107]. However, these observations do not rule out involvement of other ROS-generating systems and do not reveal if it is specific to particular cancer types. For HDACi, inhibition of the antioxidant thioredoxin has been implicated for production of ROS by HDACi [28]. Further examination into the interaction between proteasome inhibitors and HDACi may reveal additional mechanisms at play, similar to those observed by Bhalla et al. in their lymphoma studies.

The other relevant mechanism that is thought to be important in the synergistic effects between these two agents is disruption of aggresome formation. Work by Nawrocki et al. showed that bortezomib induced ubiquitin-conjugated protein aggregates, which appeared to provide a protective mechanism to cells exposed to proteasome inhibitors [108]. Studies using in vivo and in vitro pancreatic cancer models demonstrated that these cytoprotective benefits were compromised when employing vorinostat or siRNA against HDAC 6 (a cytoskeleton- associated HDAC known to be required for aggresome formation), resulting in endoplasmic reticulum (ER) stress and synergistic apoptosis [108]. This was a selective effect since neither aggresome formation by bortezomib nor apoptosis by bortezomib/vorinostat regimen was observed in normal human pancreatic epithelial cells or in murine pancreatic epithelial cells in vivo. These observations supported earlier work in MM cells using the specific HDAC 6 inhibitor, tubacin, which synergized with 
Proteasome inhibitors

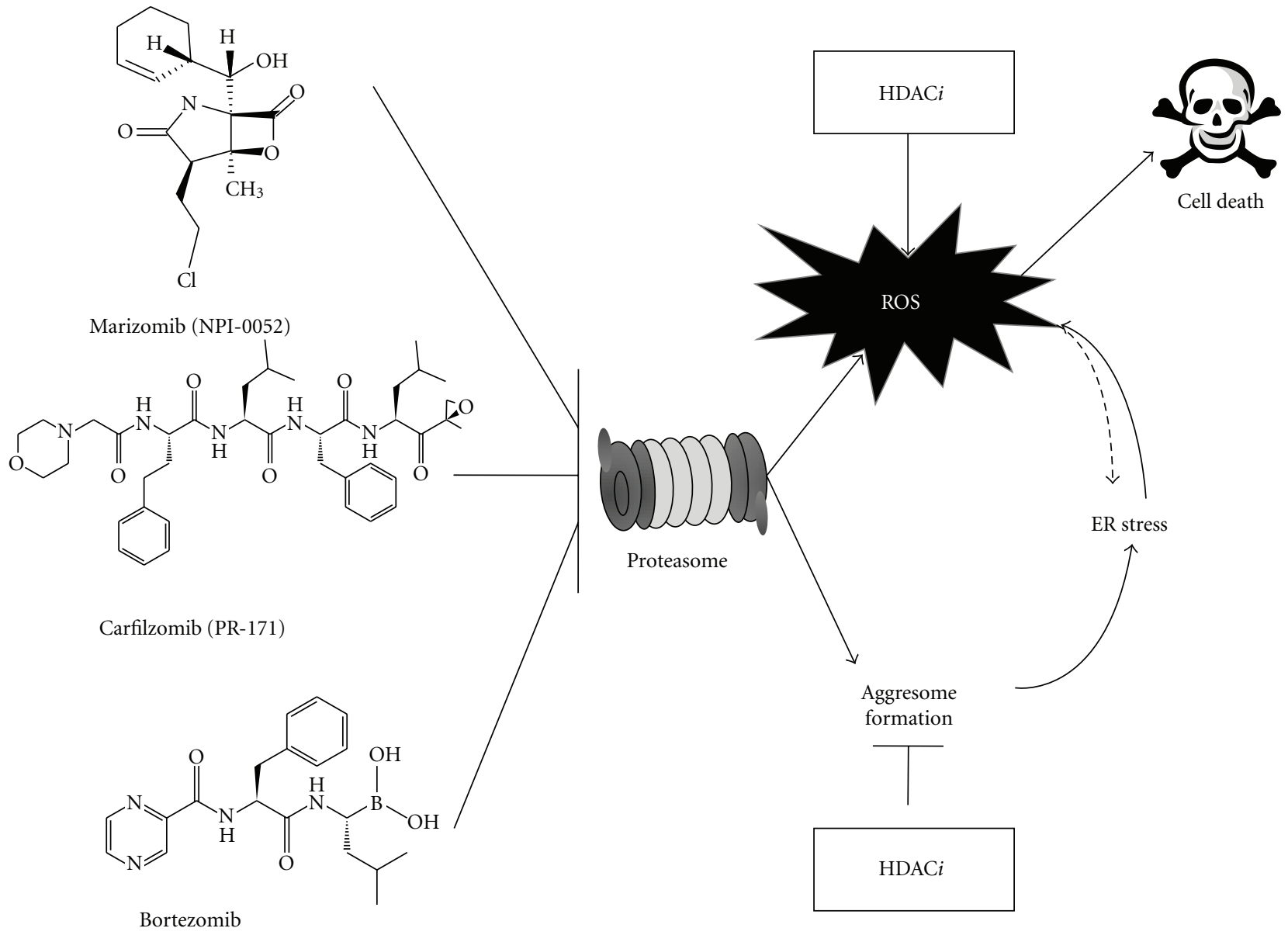

FIGURE 3: Combination of HDACi with proteasome inhibitors induces cellular stress. Synergistic apoptosis is observed between HDACi and three structurally different proteasome inhibitors-marizomib, carfilzomib, and bortezomib. The cell death observed with this therapeutic strategy is generally oxidant dependent. Individually both proteasome inhibitors and HDACi generate ROS, either via mitochondrial injury or by disregulating antioxidant systems as described in the text. When paired, these two compounds dramatically increase oxidative stress, which leads to apoptosis. High levels of ROS can also cause damage to proteins which can contribute to ER stress. Inhibiting the proteasome also results in aggregates of conjugated ubiquitin proteins that were originally to be degraded by the proteolytic complex. HDAC 6 mediates aggresome formation as a cytoprotective measure in the cell. Addition of HDACi disrupts aggresomes, leading to ER stress, which can stimulate oxidative stress or directly induce apoptosis.

bortezomib to induce lethality [109]. Both oxidative stress and interfering with aggresome formation leading to induction of ER stress are important pathways described that are considered to contribute to the synergy observed between bortezomib and HDACi. However, since both of these drugs have many pleiotropic effects, one cannot discard other mechanisms also being involved. Nevertheless, the preclinical evidence demonstrating synergy between these compounds warrants studying this combination in patients. Indeed, combination therapy between bortezomib and HDACi is currently being evaluated in numerous clinical trials (Table 1).

8.2. Marizomib. Marizomib is a clinically relevant naturally derived proteasome inhibitor that has been shown to block all three enzymatic activities of the proteasome resulting in programmed cell death in leukemic, MM, Waldenstrom's macroglobulinemia, colorectal, and pancreatic cancer cells [110-114]. The combination of marizomib and vorinostat is being evaluated in a phase I clinical trial in patients with selected advanced malignancies (Table 1). We combined this irreversible proteasome inhibitor with HDACi (vorinostat or entinostat) and showed for the first time that this regimen induced synergistic apoptosis in both primary and cultured acute leukemia cells [115]. Isobologram analysis indicated that these synergistic effects were stronger than those achieved with a bortezomib and HDACi combination. Intracellular superoxide levels were also observed with marizomib/entinostat or vorinostat treatment compared to single agents in a Jurkat ALL cell line [115]. Work in MM and our studies in leukemia had previously identified caspase- 8 as an important regulator of marizomib-induced apoptosis [110, 113]. Furthermore, we had also shown that the cytotoxicity 
observed in leukemia cells with marizomib was oxidant dependent since an antioxidant prevented apoptosis. Using a variant of the Jurkat cell line that lacked caspase-8, we confirmed the requirement of this caspase for ROS-generation by marizomib alone and in combination with HDACi [115]. Interestingly, we also observed that marizomib and $\mathrm{HDACi}$ shared overlapping biochemical effects. Both vorinostat and entinostat were able to downregulate mRNA expression levels of beta subunits that contain the proteolytic activities of the proteasome and accordingly decreased their enzymatic effects [115]. We also showed that marizomib was able to increase histone-H3 expression and acetylation [115]. This was the first report of this epigenetic alteration, usually associated with HDAC inhibition, occurring as a consequence of proteasome inhibition, and it was specific to marizomib since bortezomib did not elicit the same effect. Furthermore, caspase- 8 influenced the acetylation triggered by marizomib, since the proteasome inhibitor did not influence the acetylation in caspase-8-deficient cells, but this effect was recovered when caspase- 8 was re-expressed. Overall, our results demonstrated that caspase- 8 and oxidative stress contribute to the synergy observed between marizomib and HDACi. Unlike bortezomib, the novel second-generation proteasome inhibitor also had the ability to influence an epigenetic modification, as demonstrated by acetylation of histone $\mathrm{H} 3$, and provided another potential mechanism explaining why more synergy is observed with marizomib/HDACi compared to a bortezomib/HDACi regimen. However, a more recent study suggests that bortezomib may also be capable of modulating acetylation. In fact, their work with bortezomib may provide the missing link observed with marizomib's caspase-8-dependent acetylation. Kikuchi et al. report transcriptional downregulation of class I HDACs by bortezomib, which is mediated by caspase-8-dependent degradation of transcription factor, SP1 [116]. These data provides further evidence that proteasome inhibitors share an overlapping mechanism with HDACi.

8.3. Carfilzomib. Formerly known as PR-171, carfilzomib is a newly described irreversible proteasome inhibitor. This synthetic epoxyketone-based inhibitor is selective to potently block the chymotrypsin-like activity and is currently in clinical trials for MM and non-Hodgkin's lymphoma [117]. Compared to bortezomib, carfilzomib more potently killed MM cells and overcame resistance in patient-derived cells that demonstrated bortezomib resistance in the clinic [118]. Given that bortezomib is able to interact with HDACi to cause apoptosis, it is plausible that this next-generation proteasome inhibitor can also act synergistically with HDACi. Studies in diffuse large B cell lymphoma (DLBCL) demonstrated that carfilzomib and vorinostat interacted synergistically in DLBCL [119]. Notably, increased JNK activation contributed to lethality since interference RNA against JNK, dominant negative JNK, and peptide inhibitors reduced JNK activation and attenuated carfilzomib/HDACi cytotoxicity [119]. Combination treatment with carfilzomib and vorinostat also increased DNA damage, apoptosis and reduced tumor growth in mouse models. This regimen also showed activity against primary cells and DLBCL cells resistant to bortezomib [119].

Oxidative stress, ER stress, and JNK activation are common pathways by which proteasome inhibitors and HDACi work in concert to enhance lethality in tumor cells. However, mechanistic studies between HDACi and the next-generation proteasome inhibitors are identifying new potential mechanisms, such as acetylation by marizomib that may also be involved in triggering cytotoxicity. Given that both HDACi and proteasome inhibitors influence numerous cellular effects, further studies examining their interactions may reveal additional overlapping mechanisms that have not yet been identified as contributing to their synergism. Together, the preclinical studies between proteasome inhibitors and the epigenetic modifiers, HDACi, have provided substantial data reinforcing the potential clinical utility of these two compounds, and results from the current clinical trials are anxiously being awaited.

\section{HDACi and DNA-Damaging Agents}

Another combination that may provide synergistic benefits to cancer patients is that of HDACi with agents that cause DNA damage. HDACi have been widely shown to lead to radiosensitization in cell lines and in some mouse models, and there are many possible explanations for these effects. One idea is that histone deacetylase inhibitors are capable of disrupting the DNA damage response, so their addition prolongs the effects of DNA-damaging agents. This idea is supported by the fact that many researchers have observed prolonged $\gamma$-H2AX, which indicates the presence of DNA double-strand breaks, when HDACi are added to treatment with DNA-damaging agents such as radiation [120, 121]. Another possible explanation is that treatment with $\mathrm{HDACi}$ "loosens" the chromatin conformation, making the DNA more accessible to damaging agents. While it is difficult to provide concrete evidence for this idea, it is definitely clear that chromatin structure is an important consideration for the cell's ability to sense and repair double-strand breaks [122]. A final idea for why HDACi are effective radiosensitizers draws from the ability of HDACi to modulate gene expression, so they may act by changing expression of genes specifically involved in DNA damage responses or survival [123]. This hypothesis is supported by previous reports that HDACi lead to increases in several well-known proapoptotic proteins, such as Bim and Bmf [123]. To test these hypotheses, many HDACi have been combined with DNA-damaging agents such as radiation and chemotherapeutic agents.

9.1. Radiation. Several HDACi have shown efficacy as radiosensitizing agents when combined with ionizing radiation (IR). Many different HDACi have been tested in a wide panel of different cell lines. Some models where HDACi have been shown to radiosensitize cell lines include the use of valproic acid in colon cancer [120] and brain tumors [124], panobinostat (LBH589) in non small cell lung cancer [121], TSA in 


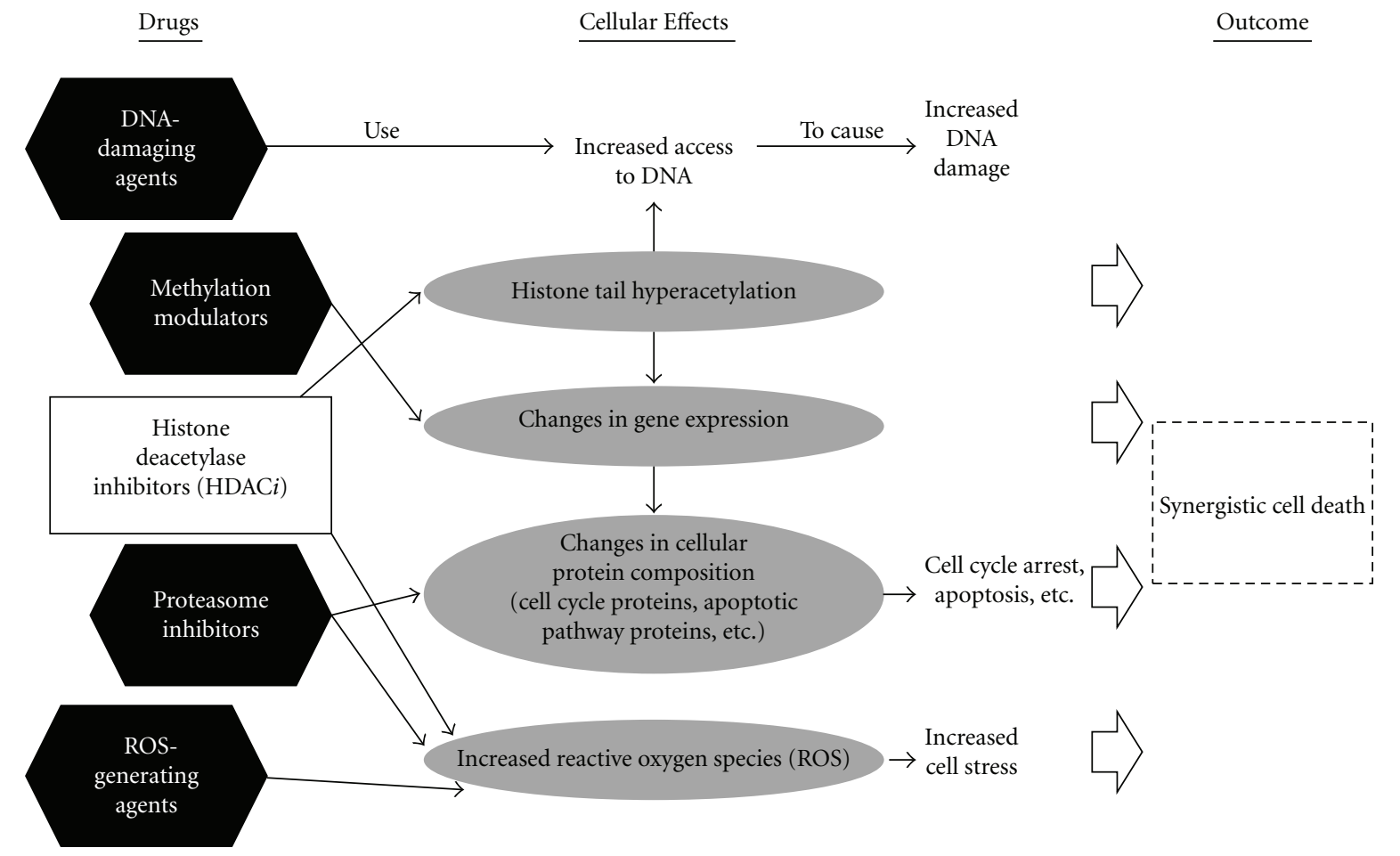

FIGURE 4: Several therapeutic combinations with HDACi show promising results. Some agents which have been shown to cause synergistic cell death when combined with HDACi include (1) DNA-damaging agents, such as radiation and many chemotherapies, which may take advantage of the increased access to DNA, provided by the "loose" chromatin arrangement after HDACi treatment, to cause increased DNA damage, (2) modulators of methylation, such as histone demethylase inhibitors and DNA methyltransferase inhibitors, which have been shown to contribute changes to the methylome that act synergistically with the actions of HDACi, (3) proteasome inhibitors, which increase cellular stress and lead to production of reactive oxygen species, can synergize with HDACi, and (4) ROS-generating agents, such as adaphostin, work with HDACi to amplify ROS production, adding to the already increased ROS levels in cancer cells until toxic levels lead to cell death.

non small cell lung cancer [125] and medulloblastoma [126], FK228 in gastric and colorectal adenocarcinoma [127], PCI24781 in cervical and colon carcinoma [128], and vorinostat in medulloblastoma [126] and melanoma [129]. There have also been studies in mouse models that indicate that this is an effective combination. Camphausen et al. have found that the treatment of DU145 prostate carcinoma xenografts with entinostat radiosensitized them in a manner that correlated with their ability to cause hyperacetylation in these animals [130]. Clearly, the evidence that HDACi can successfully sensitize cells to radiation is quickly accumulating.

Interestingly, Kim et al. have shown that inhibiting some classes of HDACs seems to be more effective than inhibiting others. This is an important observation since each HDACi has a different inhibition profile. This study showed that the most effective inhibitors were TSA (inhibits class I and II HDACs) followed by SK7041 (inhibitor of class I), while the least effective inhibitor was a suppressor of class III HDACs (splitomicin) [131].

9.2. Topoisomerase II Inhibitors. Many of the same concepts mentioned previously can be applied to combinations of HDACi with various chemotherapies that induce DNA damage. This line of thought led investigators to combine HDACi with topoisomerase II (Topo II) inhibitors. In a study where several cancer cell lines were treated with either vorinostat or TSA, then subsequently treated with a Topo II inhibitor such as cisplatin or 5 -fluorouracil, the combination was shown to be more effective at causing cell death than the chemotherapeutics alone. The investigators in this study also tried to switch the drug order, treating with the chemotherapy first and the HDACi second. This particular administration did not offer any sensitivity advantage over using the chemotherapy as a single agent. The authors proposed that this was evidence that the effectiveness of the combination stems from the ability of HDACi to allow increased access to DNA to the Topo II enzymes, which are then "locked" onto the DNA by the subsequent treatment with Topo II inhibitors, leading to increased DNA damage [132].

9.3. Temozolomide. Temozolomide is an alkylating agent that is commonly combined with radiation for the treatment of gliomas [133]. A study in a glioblastoma cell line showed that the best response may be seen when the HDACi AN-9 was combined with both radiation and temozolomide [134]. Observations of the effectiveness of temozolomide with HDACi have led to several clinical trials, mainly for gliomas (http://www.clinicaltrials.gov/). Though trials are mostly still in early stages, preliminary results indicate that the side 
effects of combinations such as vorinostat and temozolomide are fairly well tolerated [135]. A few of the combinations of HDACi with temozolomide now in clinical trials can be found in Table 1.

Overall, studies examining the interaction between HDACi and DNA-damaging agents, including radiation or chemotherapeutics, are providing the rationale to combine to these anticancer therapies for clinical utility.

\section{Conclusions}

Inhibitors of histone deacetylases are promising compounds for the treatment of cancer, and as a result, the first HDACi was approved for CTCL. Many of the cellular outcomes of HDAC inhibition are caused by changes in gene expression that influence growth inhibition, differentiation, and apoptosis of malignant cells. However, due to their limited activity in specific cancer types as single agents, the future of HDACi may reside in combination therapies. Here, we reviewed potential candidates, such as inhibitors of DNA methyltransferases and histone demethylases, ROSgenerating compounds, proteasome inhibitors, and DNAdamaging agents, that have demonstrated enhanced efficacy when combined with HDACi and may provide a therapeutic advantage in the clinic (Figure 4). These combination studies offer the rationale to explore these therapies and provide the molecular framework for better therapeutic strategies. Preclinical data suggests that combination treatments may lead to better efficacy and utility of HDACi in the clinic. However, the effects of these combinations on dose-limiting toxicities have not thoroughly been evaluated. Combination studies will offer the opportunity to use lower doses and reduce dose-limiting toxicities, which include fatigue, vomiting, nausea, and diarrhea, among others, that have been observed with HDACi as single agents [39, 45, 50]. While there could be concerns that using combinations of agents may result in increased toxicity, the preliminary data from current clinical trials show promise that combinations can be safe and tolerated. Early data from phase I studies in refractory or relapsed multiple myeloma patients using HDACi/bortezomib regimens indicate similar adverse effects associated with HDACi but no dose-limiting toxicities have yet to be reported [136]. In another ongoing study evaluating vorinostat with 5 -azacitidine in a phase I trial in myelodysplastic syndrome $(n=20)$ and acute myeloid leukemias $(n=8)$ patients, preliminary results demonstrate complete response in $43 \%$ of participants, while observing grade 1-2 toxicities (fatigue and anorexia) [137]. There are some regimens that are showing response but present toxicities [138]; however, these combinations are currently being tested in phase I trials where the main objective is to try to determine the ideal doses that will improve efficacy with minimal toxicities to patients. Additionally, another important factor to consider is that most participants enrolled in clinical trials have already been exposed to various anticancer agents and chemotherapeutics and already present preexisting conditions that perhaps influence their sensitivity to dose-limiting toxicities. It will not be until these ongoing trials are complete that we can make a definitive conclusion whether these combination approaches are suitable alternatives for cancer patients. While HDACi are providing anticancer benefits, we cannot exclude that the precise mode of action of how HDACi are killing cancer cells is still unknown. Thus, further understanding of this process may offer insights into how best to use these inhibitors and design better treatment regimens. Much of the work to date focuses on examining gene alterations as a consequence of HDAC inhibition due to acetylation of histones; however, new exciting data seems to suggest that we have to take into consideration other mechanisms. Interestingly, hyperacetylation of nonhistone proteins is linked to the HDACi effect on cancer cells [139]. Transcription factors such as p53, HIF-1, and E2F1, signaling molecule Smad 7, the chaperone protein Hsp 90, and the structural protein tubulin are all substrates of HDACs. A recent study examining the acetylome identified numerous target proteins that can be acetylated. A mass spectrometry study identified over 3,500 lysine acetylation sites in 1,750 proteins [51]. Importantly, they observed an increase in the acetylation of nonhistone proteins in cells treated with HDACi, entinostat and vorinostat. Surprisingly, these HDACi only increase acetylation on $10 \%$ of all acetylation sites suggesting that their effects are very selective. As expected, differences in acetylation of substrates were observed between both HDACi. For example, vorinostat was a more potent inducer of histone acetylation and of DNA double-strand breaks, as demonstrated by an increase in $\gamma$ H2AX. The nonhistone substrate, Hsp90, was also highly acetylated with vorinostat but not with entinostat. On the other hand, the tumor suppressor p53 was acetylated to a higher degree with entinostat whereas vorinostat did not influence the acetylation of this protein. These differences probably reflect the ability of these compounds to target different HDACs. The identification of the acetylome offers a snapshot of the different proteins that may be influenced by acetylation and therefore by HDACi. These types of experiments that attempt to elucidate the great spectrum of protein acetylation [51] have revealed new targets and unknown potential mechanisms by which HDACi may work as an effective therapy for cancer. Studies like these can be mined to identify molecular end points that can be targeted with more efficacious HDACi-combination therapies.

\section{References}

[1] L. Hong, G. P. Schroth, H. R. Matthews, P. Yau, and E. M. Bradbury, "Studies of the DNA binding properties of histone H4 amino terminus. Thermal denaturation studies reveal that acetylation markedly reduces the binding constant of the H4 'tail' to DNA," Journal of Biological Chemistry, vol. 268, no. 1, pp. 305-314, 1993.

[2] V. G. Allfrey, R. Faulkner, and A. E. Mirsky, "Acetylation and methylation of histones and their possible role in the regulation of RNA synthesis," Proceedings of the National Academy of Sciences of the United States of, vol. 51, pp. 786794, 1964.

[3] B. D. Strahl and C. D. Allis, "The language of covalent histone modifications," Nature, vol. 403, no. 6765, pp. 41-45, 2000. 
[4] L. Gao, M. A. Cueto, F. Asselbergs, and P. Atadja, "Cloning and functional characterization of HDAC11, a novel member of the human histone deacetylase family," Journal of Biological Chemistry, vol. 277, no. 28, pp. 25748-25755, 2002.

[5] S. I. Imai and L. Guarente, "Ten years of NAD-dependent SIR2 family deacetylases: implications for metabolic diseases," Trends in Pharmacological Sciences, vol. 31, no. 5, pp. 212-220, 2010.

[6] W. Gu and R. G. Roeder, "Activation of p53 sequence-specific DNA binding by acetylation of the p53 C-terminal domain," Cell, vol. 90, no. 4, pp. 595-606, 1997.

[7] K. B. Glaser, J. Li, M. J. Staver, R. Q. Wei, D. H. Albert, and S. K. Davidsen, "Role of Class I and Class II histone deacetylases in carcinoma cells using siRNA," Biochemical and Biophysical Research Communications, vol. 310, no. 2, pp. 529-536, 2003.

[8] P. Atadja, L. Gao, P. Kwon et al., "Selective growth inhibition of tumor cells by a novel histone deacetylase inhibitor, NVPLAQ824," Cancer Research, vol. 64, no. 2, pp. 689-695, 2004.

[9] J. S. Ungerstedt, Y. Sowa, W. S. Xu et al., "Role of thioredoxin in the response of normal and transformed cells to histone deacetylase inhibitors," Proceedings of the National Academy of Sciences of the United States of America, vol. 102, no. 3, pp. 673-678, 2005.

[10] A. Nebbioso, N. Clarke, E. Voltz et al., "Tumor-selective action of HDAC inhibitors involves TRAIL induction in acute myeloid leukemia cells," Nature Medicine, vol. 11, no. 1, pp. 77-84, 2005.

[11] R. W. Johnstone, "Histone-deacetylase inhibitors: novel drugs for the treatment of cancer," Nature Reviews Drug Discovery, vol. 1, no. 4, pp. 287-299, 2002.

[12] C. Friend, W. Scher, J. G. Holland, and T. Sato, "Hemoglobin synthesis in murine virus-induced leukemic cells in vitro: stimulation of erythroid differentiation by dimethyl sulfoxide," Proceedings of the National Academy of Sciences of the United States of America, vol. 68, no. 2, pp. 378-382, 1971.

[13] M. Yoshida, M. Kijima, M. Akita, and T. Beppu, "Potent and specific inhibition of mammalian histone deacetylase both in vivo and in vitro by trichostatin A," Journal of Biological Chemistry, vol. 265, no. 28, pp. 17174-17179, 1990.

[14] V. M. Richon, R. G. Ramsay, R. A. Rifkind, and P. A. Marks, "Modulation of the c-myb, c-myc and p53 mRNA and protein levels during induced murine erythroleukemia cell differentiation," Oncogene, vol. 4, no. 2, pp. 165-173, 1989.

[15] V. M. Richon, S. Emiliani, E. Verdin et al., "A class of hybrid polar inducers of transformed cell differentiation inhibits histone deacetylases," Proceedings of the National Academy of Sciences of the United States of America, vol. 95, no. 6, pp. 3003-3007, 1998.

[16] S. J. Haggarty, K. M. Koeller, J. C. Wong, C. M. Grozinger, and S. L. Schreiber, "Domain-selective small-molecule inhibitor of histone deacetylase 6 (HDAC6)-mediated tubulin deacetylation," Proceedings of the National Academy of Sciences of the United States of America, vol. 100, no. 8, pp. 4389-4394, 2003.

[17] J. J. Buggy, Z. A. Cao, K. E. Bass et al., "CRA-024781: a novel synthetic inhibitor of histone deacetylase enzymes with antitumor activity in vitro and in vivo," Molecular Cancer Therapeutics, vol. 5, no. 5, pp. 1309-1317, 2006.

[18] J. M. Mariadason, G. A. Corner, and L. H. Augenlicht, "Genetic reprogramming in pathways of colonic cell maturation induced by short chain fatty acids: comparison with trichostatin A, sulindac, and curcumin and implications for chemoprevention of colon cancer," Cancer Research, vol. 60, no. 16, pp. 4561-4572, 2000.
[19] S. Minucci and P. G. Pelicci, "Histone deacetylase inhibitors and the promise of epigenetic (and more) treatments for cancer," Nature Reviews Cancer, vol. 6, no. 1, pp. 38-51, 2006.

[20] S. H. Kwon, S. H. Ahn, Y. K. Kim et al., "Apicidin, a histone deacetylase inhibitor, induces apoptosis and Fas/Fas ligand expression in human acute promyelocytic leukemia cells," Journal of Biological Chemistry, vol. 277, no. 3, pp. 20732080, 2002.

[21] A. Insinga, S. Monestiroli, S. Ronzoni et al., "Inhibitors of histone deacetylases induce tumor-selective apoptosis through activation of the death receptor pathway," Nature Medicine, vol. 11, no. 1, pp. 71-76, 2005.

[22] J. A. Vrana, R. H. Decker, C. R. Johnson et al., "Induction of apoptosis in U937 human leukemia cells by suberoylanilide hydroxamic acid (SAHA) proceeds through pathways that are regulated by $\mathrm{Bcl}-2 / \mathrm{Bcl}-\mathrm{x}(\mathrm{L}), \mathrm{c}-J u n$, and $\mathrm{p} 21(\mathrm{CIP} 1)$, but independent of p53," Oncogene, vol. 18, no. 50, pp. 70167025, 1999.

[23] Y. Zhao, J. Tan, L. Zhuang, X. Jiang, E. T. Liu, and Q. Yu, "Inhibitors of histone deacetylases target the RbE2F1 pathway for apoptosis induction through activation of proapoptotic protein Bim," Proceedings of the National Academy of Sciences of the United States of America, vol. 102, no. 44, pp. 16090-16095, 2005.

[24] A. A. Ruefli, M. J. Ausserlechner, D. Bernhard et al., "The histone deacetylase inhibitor and chemotherapeutic agent suberoylanilide hydroxamic acid (SAHA) induces a cell-death pathway characterized by cleavage of Bid and production of reactive oxygen species," Proceedings of the National Academy of Sciences of the United States of America, vol. 98, no. 19, pp. 10833-10838, 2001.

[25] R. R. Rosato, J. A. Almenara, S. C. Maggio et al., "Role of histone deacetylase inhibitor-induced reactive oxygen species and DNA damage in LAQ-824/fludarabine antileukemic interactions," Molecular Cancer Therapeutics, vol. 7, no. 10, pp. 3285-3297, 2008.

[26] S. Gao, A. Mobley, C. Miller, J. Boklan, and J. Chandra, "Potentiation of reactive oxygen species is a marker for synergistic cytotoxicity of MS-275 and 5-azacytidine in leukemic cells," Leukemia Research, vol. 32, no. 5, pp. 771780, 2008.

[27] R. R. Rosato, J. A. Almenara, and S. Grant, "The histone deacetylase inhibitor MS-275 promotes differentiation or apoptosis in human leukemia cells through a process regulated by generation of reactive oxygen species and induction of p21," Cancer Research, vol. 63, no. 13, pp. 3637-3645, 2003.

[28] L. M. Butler, X. Zhou, W. S. Xu et al., "The histone deacetylase inhibitor SAHA arrests cancer cell growth, upregulates thioredoxin-binding protein-2, and down-regulates thioredoxin," Proceedings of the National Academy of Sciences of the United States of America, vol. 99, no. 18, pp. 1170011705, 2002.

[29] D. T. Lincoln, E. M. Ali Emadi, K. F. Tonissen, and F. M. Clarke, "The thioredoxin-thioredoxin reductase system: over-expression in human cancer," Anticancer Research, vol. 23, no. 3 B, pp. 2425-2433, 2003.

[30] L. E. Shao, M. B. Diccianni, T. Tanaka et al., "Thioredoxin expression in primary T-cell acute lymphoblastic leukemia and its therapeutic implication," Cancer Research, vol. 61, no. 19, pp. 7333-7338, 2001.

[31] V. M. Richon, T. W. Sandhoff, R. A. Rifkind, and P. A. Marks, "Histone deacetylase inhibitor selectively induces p21 expressjon and gene-associated histone acetylation," 
Proceedings of the National Academy of Sciences of the United States of America, vol. 97, no. 18, pp. 10014-10019, 2000.

[32] L. Qiu, A. Burgess, D. P. Fairlie, H. Leonard, P. G. Parsons, and B. G. Gabrielli, "Histone deacetylase inhibitors trigger a G2 checkpoint in normal cells that is defective in tumor cells," Molecular Biology of the Cell, vol. 11, no. 6, pp. 2069-2083, 2000.

[33] V. Sandor, A. Senderowicz, S. Mertins et al., "P21-dependent $\mathrm{G}$ arrest with downregulation of cyclin D1 and upregulation of cyclin E by the histone deacetylase inhibitor FR901228," British Journal of Cancer, vol. 83, no. 6, pp. 817-825, 2000.

[34] D. Z. Qian, Y. Kato, S. Shabbeer et al., "Targeting tumor angiogenesis with histone deacetylase inhibitors: the hydroxamic acid derivative LBH589," Clinical Cancer Research, vol. 12, no. 2, pp. 634-642, 2006.

[35] C. F. Deroanne, K. Bonjean, S. Servotte et al., "Histone deacetylases inhibitors as anti-angiogenic agents altering vascular endothelial growth factor signaling," Oncogene, vol. 21, no. 3, pp. 427-436, 2002.

[36] M. Michaelis, U. R. Michaelis, I. Fleming et al., "Valproic acid inhibits angiogenesis in vitro and in vivo," Molecular Pharmacology, vol. 65, no. 3, pp. 520-527, 2004.

[37] M. Duvic, R. Talpur, X. Ni et al., "Phase 2 trial of oral vorinostat (suberoylanilide hydroxamic acid, SAHA) for refractory cutaneous T-cell lymphoma (CTCL)," Blood, vol. 109, no. 1, pp. 31-39, 2007.

[38] S. C. Modesitt, M. Sill, J. S. Hoffman, and D. P. Bender, "A phase II study of vorinostat in the treatment of persistent or recurrent epithelial ovarian or primary peritoneal carcinoma: a Gynecologic Oncology Group study," Gynecologic Oncology, vol. 109, no. 2, pp. 182-186, 2008.

[39] J. Vansteenkiste, E. Van Cutsem, H. Dumez et al., "Early phase II trial of oral vorinostat in relapsed or refractory breast, colorectal, or non-small cell lung cancer," Investigational New Drugs, vol. 26, no. 5, pp. 483-488, 2008.

[40] G. R. Blumenschein, M. S. Kies, V. A. Papadimitrakopoulou et al., "Phase II trial of the histone deacetylase inhibitor vorinostat $\left(Z_{\text {Zolinza }}{ }^{\mathrm{TM}}\right.$, suberoylanilide hydroxamic acid, SAHA) in patients with recurrent and/or metastatic head and neck cancer," Investigational New Drugs, vol. 26, no. 1, pp. 8187,2008 .

[41] E. Galanis, K. A. Jaeckle, M. J. Maurer et al., "Phase II trial of Vorinostat in recurrent glioblastoma multiforme: a north central cancer treatment group study," Journal of Clinical Oncology, vol. 27, no. 12, pp. 2052-2058, 2009.

[42] T. H. Luu, R. J. Morgan, L. Leong et al., "A phase II trial of vorinostat (suberoylanilide hydroxamic acid) in metastatic breast cancer: a California cancer consortium study," Clinical Cancer Research, vol. 14, no. 21, pp. 7138-7142, 2008.

[43] J. A. Woyach, R. T. Kloos, M. D. Ringel et al., "Lack of therapeutic effect of the histone deacetylase inhibitor vorinostat in patients with metastatic radioiodine-refractory thyroid carcinoma," Journal of Clinical Endocrinology and Metabolism, vol. 94, no. 1, pp. 164-170, 2009.

[44] X. Ma, H. H. Ezzeldin, and R. B. Diasio, "Histone deacetylase inhibitors: current status and overview of recent clinical trials," Drugs, vol. 69, no. 14, pp. 1911-1934, 2009.

[45] I. Gojo, A. Jiemjit, J. B. Trepel et al., "Phase 1 and pharmacologic study of MS-275, a histone deacetylase inhibitor, in adults with refractory and relapsed acute leukemias," Blood, vol. 109, no. 7, pp. 2781-2790, 2007.

[46] Q. C. Ryan, D. Headlee, M. Acharya et al., "Phase I and pharmacokinetic study of MS-275, a histone deacetylase inhibitor, in patients with advanced and refractory solid tumors or lymphoma," Journal of Clinical Oncology, vol. 23, no. 17, pp. 3912-3922, 2005.

[47] S. Kummar, M. Gutierrez, E. R. Gardner et al., "Phase I trial of MS-275, a histone deacetylase inhibitor, administered weekly in refractory solid tumors and lymphoid malignancies," Clinical Cancer Research, vol. 13, no. 18, pp. 5411-5417, 2007.

[48] L. Gore, M. L. Rothenberg, C. L. O’Bryant et al., "A phase I and pharmacokinetic study of the oral histone deacetylase inhibitor, MS-275, in patients with refractory solid tumors and lymphomas," Clinical Cancer Research, vol. 14, no. 14, pp. 4517-4525, 2008.

[49] O. A. O’Connor, M. L. Heaney, L. Schwartz et al., "Clinical experience with intravenous and oral formulations of the novel histone deacetylase inhibitor suberoylanilide hydroxamic acid in patients with advanced hematologic malignancies," Journal of Clinical Oncology, vol. 24, no. 1, pp. 166-173, 2006.

[50] W. K. Kelly, O. A. O’Connor, L. M. Krug et al., "Phase I study of an oral histone deacetylase inhibitor, suberoylanilide hydroxamic acid, in patients with advanced cancer," Journal of Clinical Oncology, vol. 23, no. 17, pp. 3923-3931, 2005.

[51] C. Choudhary, C. Kumar, F. Gnad et al., "Lysine acetylation targets protein complexes and co-regulates major cellular functions," Science, vol. 325, no. 5942, pp. 834-840, 2009.

[52] A. Bird, "DNA methylation patterns and epigenetic memory," Genes and Development, vol. 16, no. 1, pp. 6-21, 2002.

[53] P. H. Tate and A. P. Bird, "Effects of DNA methylation on DNA-binding proteins and gene expression," Current Opinion in Genetics and Development, vol. 3, no. 2, pp. 226231, 1993.

[54] R. R. Meehan, J. D. Lewis, S. McKay, E. L. Kleiner, and A. P. Bird, "Identification of a mammalian protein that binds specifically to DNA containing methylated CpGs," Cell, vol. 58, no. 3, pp. 499-507, 1989.

[55] B. Hendrich and A. Bird, "Identification and characterization of a family of mammalian methyl-CpG binding proteins," Molecular and Cellular Biology, vol. 18, no. 11, pp. 6538-6547, 1998.

[56] P. L. Jones, G. J. C. Veenstra, P. A. Wade et al., "Methylated DNA and MeCP2 recruit histone deacetylase to repress transcription," Nature Genetics, vol. 19, no. 2, pp. 187-191, 1998.

[57] X. Nan, H. H. Ng, C. A. Johnson et al., "Transcriptional repression by the methyl-CpG-binding protein $\mathrm{MeCP} 2$ involves a histone deacetylase complex," Nature, vol. 393, no. 6683, pp. 386-389, 1998.

[58] A. Dobrovic and D. Simpfendorfer, "Methylation of the BRCA1 gene in sporadic breast cancer," Cancer Research, vol. 57, no. 16, pp. 3347-3350, 1997.

[59] V. Greger, E. Passarge, W. Hopping, E. Messmer, and B. Horsthemke, "Epigenetic changes may contribute to the formation and spontaneous regression of retinoblastoma," Human Genetics, vol. 83, no. 2, pp. 155-158, 1989.

[60] M. Esteller, S. R. Hamilton, P. C. Burger, S. B. Baylin, and J. G. Herman, "Inactivation of the DNA repair gene $\mathrm{O}(6)$-methylguanine-DNA methyltransferase by promoter hypermethylation is a common event in primary human neoplasia," Cancer Research, vol. 59, no. 4, pp. 793-797, 1999.

[61] LI. Ding, L. Qiu, J. Zhang, and B. Guo, "Camptothecin-induced cell proliferation inhibition and apoptosis enhanced 
by DNA methyltransferase inhibitor, 5-aza-2'-deoxycytidine," Biological and Pharmaceutical Bulletin, vol. 32, no. 6, pp. 1105-1108, 2009.

[62] A. Merlo, J. G. Herman, L. Mao et al., “5” CpG island methylation is associated with transcriptional silencing of the tumour suppressor p16/CDKN2/MTS1 in human cancers," Nature Medicine, vol. 1, no. 7, pp. 686-692, 1995.

[63] G. Deng, A. Chen, J. Hong, H. S. Chae, and Y. S. Kim, "Methylation of CpG in a small region of the hMLH1 promoter invariably correlates with the absence of gene expression," Cancer Research, vol. 59, no. 9, pp. 2029-2033, 1999.

[64] D. Gius, H. Cui, C. M. Bradbury et al., "Distinct effects on gene expression of chemical and genetic manipulation of the cancer epigenome revealed by a multimodality approach," Cancer Cell, vol. 6, no. 4, pp. 361-371, 2004.

[65] T. J. Walton, G. Li, R. Seth, S. E. McArdle, M. C. Bishop, and R. C. Rees, "DNA demethylation and histone deacetylation inhibition co-operate to re-express estrogen receptor beta and induce apoptosis in prostate cancer cell-lines," Prostate, vol. 68, no. 2, pp. 210-222, 2008.

[66] D. Cecconi, M. Donadelli, E. D. Pozza et al., "Synergistic effect of trichostatin A and 5-aza-2'-deoxycytidine on growth inhibition of pancreatic endocrine tumour cell lines: a proteomic study," Proteomics, vol. 9, no. 7, pp. 1952-1966, 2009.

[67] M. I. Klisovic, E. A. Maghraby, M. R. Parthun et al., "Depsipeptide (FR 901228) promotes histone acetylation, gene transcription, apoptosis and its activity is enhanced by DNA methyltransferase inhibitors in AML1/ETO-positive leukemic cells," Leukemia, vol. 17, no. 2, pp. 350-358, 2003.

[68] G. Chai, L. Li, W. Zhou et al., "HDAC inhibitors act with 5 -aza-2'-deoxycytidine to inhibit cell proliferation by suppressing removal of incorporated abases in lung cancer cells," PLoS One, vol. 3, no. 6, Article ID e2445, 2008.

[69] M. A. Rudek, M. Zhao, P. He et al., "Pharmacokinetics of 5 -azacitidine administered with phenylbutyrate in patients with refractory solid tumors or hematologic malignancies," Journal of Clinical Oncology, vol. 23, no. 17, pp. 3906-3911, 2005.

[70] E. A. Griffiths and S. D. Gore, "DNA methyltransferase and histone deacetylase inhibitors in the treatment of myelodysplastic syndromes," Seminars in Hematology, vol. 45, no. 1, pp. 23-30, 2008.

[71] Y. Shi, F. Lan, C. Matson et al., "Histone demethylation mediated by the nuclear amine oxidase homolog LSD1," Cell, vol. 119, no. 7, pp. 941-953, 2004.

[72] Y. I. Tsukada, J. Fang, H. Erdjument-Bromage et al., "Histone demethylation by a family of JmjC domain-containing proteins," Nature, vol. 439, no. 7078, pp. 811-816, 2006.

[73] S. G. Gray, A. H. Iglesias, F. Lizcano et al., "Functional characterization of JMJD2A, a histone deacetylase- and retinoblastoma-binding protein," Journal of Biological Chemistry, vol. 280, no. 31, pp. 28507-28518, 2005.

[74] M. G. Lee, C. Wynder, D. A. Bochar, M. A. Hakimi, N. Cooch, and R. Shiekhattar, "Functional interplay between histone demethylase and deacetylase enzymes," Molecular and Cellular Biology, vol. 26, no. 17, pp. 6395-6402, 2006.

[75] D. M. Z. Schmidt and D. G. McCafferty, "trans-2-phenylcyclopropylamine is a mechanism-based inactivator of the histone demethylase LSD1," Biochemistry, vol. 46, no. 14, pp. 4408-4416, 2007.
[76] J. C. Culhane, L. M. Szewczuk, X. Liu, G. Da, R. Marmorstein, and P. A. Cole, "A mechanism-based inactivator for histone demethylase LSD1," Journal of the American Chemical Society, vol. 128, no. 14, pp. 4536-4537, 2006.

[77] D. M. Gooden, D. M. Z. Schmidt, J. A. Pollock, A. M. Kabadi, and D. G. McCafferty, "Facile synthesis of substituted trans2-arylcyclopropylamine inhibitors of the human histone demethylase LSD1 and monoamine oxidases A and B," Bioorganic and Medicinal Chemistry Letters, vol. 18, no. 10, pp. 3047-3051, 2008.

[78] M. G. Lee, C. Wynder, D. M. Schmidt, D. G. McCafferty, and R. Shiekhattar, "Histone H3 lysine 4 demethylation is a target of nonselective antidepressive medications," Chemistry and Biology, vol. 13, no. 6, pp. 563-567, 2006.

[79] M. Yang, J. C. Culhane, L. M. Szewczuk et al., "Structural basis for the inhibition of the LSD1 histone demethylase by the antidepressant trans-2-phenylcyclopropylamine," Biochemistry, vol. 46, no. 27, pp. 8058-8065, 2007.

[80] M. M. Singh, C. A. Manton, K. P. Bhat et al., "Inhibition of LSD1 sensitizes glioblastoma cells to histone deacetylase inhibitors," Neuro-Oncology. In press.

[81] J. Wang, S. Hevi, J. K. Kurash et al., "The lysine demethylase LSD1 (KDM1) is required for maintenance of global DNA methylation," Nature Genetics, vol. 41, no. 1, pp. 125-129, 2009.

[82] D. N. Ciccone, H. Su, S. Hevi et al., "KDM1B is a histone H3K4 demethylase required to establish maternal genomic imprints," Nature, vol. 461, no. 7262, pp. 415-418, 2009.

[83] Y. Huang, T. M. Stewart, Y. Wu et al., "Novel oligoamine analogues inhibit lysine-specific demethylase 1 and induce reexpression of epigenetically silenced genes," Clinical Cancer Research, vol. 15, no. 23, pp. 7217-7228, 2009.

[84] M. Sakurai, N. R. Rose, L. Schultz et al., "A miniaturized screen for inhibitors of Jumonji histone demethylases," Molecular BioSystems, vol. 6, no. 2, pp. 357-364, 2010.

[85] J. Chandra, "Oxidative Stress by targeted agents promotes cytotoxicity in hematologic malignancies," Antioxidants and Redox Signaling, vol. 11, no. 5, pp. 1123-1127, 2009.

[86] H. Pelicano, D. Carney, and P. Huang, "ROS stress in cancer cells and therapeutic implications," Drug Resistance Updates, vol. 7, no. 2, pp. 97-110, 2004.

[87] S. Toyokuni, "Oxidative stress and cancer: the role of redox regulation,” Biotherapy, vol. 11, no. 2-3, pp. 147-154, 1998.

[88] G. Kaur, V. L. Narayanan, P. A. Risbood et al., "Synthesis, structure-activity relationship, and p210 protein tyrosine kinase activity of novel AG 957 analogs," Bioorganic and Medicinal Chemistry, vol. 13, no. 5, pp. 1749-1761, 2005.

[89] J. Chandra, J. Tracy, D. Loegering et al., "Adaphostin-induced oxidative stress overcomes BCR/ABL mutation-dependent and -independent imatinib resistance," Blood, vol. 107, no. 6, pp. 2501-2506, 2006.

[90] J. Chandra, J. Hackbarth, S. Le et al., "Involvement of reactive oxygen species in adaphostin-induced cytotoxicity in human leukemia cells," Blood, vol. 102, no. 13, pp. 4512-4519, 2003.

[91] S. B. Le, M. K. Hailer, S. Buhrow et al., "Inhibition of mitochondrial respiration as a source of adaphostin-induced reactive oxygen species and cytotoxicity," Journal of Biological Chemistry, vol. 282, no. 12, pp. 8860-8872, 2007.

[92] L. H. Stockwin, M. A. Bumke, S. X. Yu et al., "Proteomic analysis identifies oxidative stress induction by adaphostin," Clinical Cancer Research, vol. 13, no. 12, pp. 3667-3681, 2007.

[93] K. L. Cheung and A. N. Kong, "Molecular targets of dietary phenethyl isothiocyanate and sulforaphane for cancer 
chemoprevention," AAPS Journal, vol. 12, no. 1, pp. 87-97, 2010.

[94] D. Xiao, C. S. Jonhson, D. L. Trump, and S. V. Singh, "Proteasome-mediated degradation of cell division cycle 25C and cyclin-dependent kinase 1 in phenethyl isothiocyanateinduced G-M-phase cell cycle arrest in PC-3 human prostate cancer cells," Molecular Cancer Therapeutics, vol. 3, no. 5, pp. 567-575, 2004.

[95] A. A. Beklemisheva, J. Feng, Y. A. Yeh, L. G. Wang, and J. W. Chiao, "Modulating testosterone stimulated prostate growth by phenethyl isothiocyanate via Sp1 and androgen receptor down-regulation," Prostate, vol. 67, no. 8, pp. 863-870, 2007.

[96] D. Xiao, A. A. Powolny, M. B. Moura et al., "Phenethyl isothiocyanate inhibits oxidative phosphorylation to trigger reactive oxygen species-mediated death of human prostate cancer cells," Journal of Biological Chemistry, vol. 285, no. 34, pp. 26558-26569, 2010.

[97] D. Trachootham, Y. Zhou, H. Zhang et al., "Selective killing of oncogenically transformed cells through a ROS-mediated mechanism by $\beta$-phenylethyl isothiocyanate," Cancer Cell, vol. 10, no. 3, pp. 241-252, 2006.

[98] D. Trachootham, H. Zhang, W. Zhang et al., "Effective elimination of fludarabine-resistant CLL cells by PEITC through a redox-mediated mechanism," Blood, vol. 112, no. 5, pp. 1912-1922, 2008.

[99] Y. Hu, W. Lu, G. Chen et al., "Overcoming resistance to histone deacetylase inhibitors in human leukemia with the redox modulating compound $\beta$-phenylethyl isothiocyanate," Blood, vol. 116, no. 15, pp. 2732-2741, 2010.

[100] M. Orlowski and S. Wilk, "Catalytic activities of the $20 \mathrm{~S}$ proteasome, a multicatalytic proteinase complex," Archives of Biochemistry and Biophysics, vol. 383, no. 1, pp. 1-16, 2000.

[101] P. Masdehors, S. Omura, H. Merle-Beral et al., "Increased sensitivity of CLL-derived lymphocytes to apoptotic death activation by the proteasome-specific inhibitor lactacystin," British Journal of Haematology, vol. 105, no. 3, pp. 752-757, 1999.

[102] H. C. A. Drexler, "Activation of the cell death program by inhibition of proteasome function," Proceedings of the National Academy of Sciences of the United States of America, vol. 94, no. 3, pp. 855-860, 1997.

[103] X. Y. Pei, Y. Dai, and S. Grant, "Synergistic induction of oxidative injury and apoptosis in human multiple myeloma cells by the proteasome inhibitor bortezomib and histone deacetylase inhibitors," Clinical Cancer Research, vol. 10, no. 11, pp. 3839-3852, 2004.

[104] C. Yu, M. Rahmani, D. Conrad, M. Subler, P. Dent, and S. Grant, "The proteasome inhibitor bortezomib interacts synergistically with histone deacetylase inhibitors to induce apoptosis in Bcr/Abl cells sensitive and resistant to STI571," Blood, vol. 102, no. 10, pp. 3765-3774, 2003.

[105] R. Feng, A. Oton, M. Y. Mapara, G. Anderson, C. Belani, and S. Lentzsch, "The histone deacetylase inhibitor, PXD101, potentiates bortezomib-induced anti-multiple myeloma effect by induction of oxidative stress and DNA damage," British Journal of Haematology, vol. 139, no. 3, pp. 385-397, 2007.

[106] S. Bhalla, S. Balasubramanian, K. David et al., "PCI-24781 induces caspase and reactive oxygen species-dependent apoptosis through NF- $\kappa \mathrm{B}$ mechanisms and is synergistic with bortezomib in lymphoma cells," Clinical Cancer Research, vol. 15, no. 10, pp. 3354-3365, 2009.

[107] Y. H. Ling, L. Liebes, Y. Zou, and R. Perez-Soler, "Reactive oxygen species generation and mitochondrial dysfunction in the apoptotic response to Bortezomib, a novel proteasome inhibitor, in human H460 non-small cell lung cancer cells," Journal of Biological Chemistry, vol. 278, no. 36, pp. 3371433723, 2003.

[108] S. T. Nawrocki, J. S. Carew, M. S. Pino et al., "Aggresome disruption: a novel strategy to enhance bortezomib-induced apoptosis in pancreatic cancer cells," Cancer Research, vol. 66, no. 7, pp. 3773-3781, 2006.

[109] T. Hideshima, J. E. Bradner, J. Wong et al., "Small-molecule inhibition of proteasome and aggresome function induces synergistic antitumor activity in multiple myeloma," Proceedings of the National Academy of Sciences of the United States of America, vol. 102, no. 24, pp. 8567-8572, 2005.

[110] D. Chauhan, L. Catley, G. Li et al., "A novel orally active proteasome inhibitor induces apoptosis in multiple myeloma cells with mechanisms distinct from Bortezomib," Cancer Cell, vol. 8, no. 5, pp. 407-419, 2005.

[111] S. Ruiz, Y. Krupnik, M. Keating, J. Chandra, M. Palladino, and D. McConkey, "The proteasome inhibitor NPI-0052 is a more effective inducer of apoptosis than bortezomib in lymphocytes from patients with chronic lymphocytic leukemia," Molecular Cancer Therapeutics, vol. 5, no. 7, pp. 1836-1843, 2006.

[112] J. C. Cusack, R. Liu, L. Xia et al., "NPI-0052 enhances tumoricidal response to conventional cancer therapy in a colon cancer model," Clinical Cancer Research, vol. 12, no. 22, pp. 6758-6764, 2006.

[113] C. P. Miller, K. Ban, M. E. Dujka et al., "NPI-0052, a novel proteasome inhibitor, induces caspase- 8 and ROS-dependent apoptosis alone and in combination with HDAC inhibitors in leukemia cells," Blood, vol. 110, no. 1, pp. 267-277, 2007.

[114] A. M. Roccaro, X. Leleu, A. Sacco et al., "Dual targeting of the proteasome regulates survival and homing in Waldenström macroglobulinemia," Blood, vol. 111, no. 9, pp. 4752-4763, 2008.

[115] C. P. Miller, S. Rudra, M. J. Keating, W. G. Wierda, M. Palladino, and J. Chandra, "Caspase-8 dependent histone acetylation by a novel proteasome inhibitor, NPI-0052: a mechanism for synergy in leukemia cells," Blood, vol. 113, no. 18, pp. 4289-4299, 2009.

[116] J. Kikuchi, T. Wada, R. Shimizu et al., "Histone deacetylases are critical targets of bortezomib-induced cytotoxicity in multiple myeloma," Blood, vol. 116, no. 3, pp. 406-417, 2010.

[117] O. A. O’Connor, A. K. Stewart, M. Vallone et al., "A phase 1 dose escalation study of the safety and pharmacokinetics of the novel proteasome inhibitor carfilzomib (PR-171) in patients with hematologic malignancies," Clinical Cancer Research, vol. 15, no. 22, pp. 7085-7091, 2009.

[118] D. J. Kuhn, Q. Chen, P. M. Voorhees et al., "Potent activity of carfilzomib, a novel, irreversible inhibitor of the ubiquitinproteasome pathway, against preclinical models of multiple myeloma," Blood, vol. 110, no. 9, pp. 3281-3290, 2007.

[119] G. Dasmahapatra, D. Lembersky, L. Kramer et al., "The panHDAC inhibitor vorinostat potentiates the activity of the proteasome inhibitor carfilzomib in human DLBCL cells in vitro and in vivo," Blood, vol. 115, no. 22, pp. 4478-4487, 2010.

[120] X. Chen, P. Wong, E. Radany, and J. Y. C. Wong, "HDAC inhibitor, valproic acid, induces p53-dependent radiosensitization of colon cancer cells," Cancer Biotherapy and Radiopharmaceuticals, vol. 24, no. 6, pp. 689-699, 2009. 
[121] L. Geng, K. C. Cuneo, A. Fu, T. Tu, P. W. Atadja, and D. E. Hallahan, "Histone deacetylase (HDAC) inhibitor LBH589 increases duration of $\gamma$-H2AX foci and confines HDAC4 to the cytoplasm in irradiated non-small cell lung cancer," Cancer Research, vol. 66, no. 23, pp. 11298-11304, 2006.

[122] A. R. Venkitaraman, "Modifying chromatin architecture during the response to DNA breakage," Critical Reviews in Biochemistry and Molecular Biology, vol. 45, no. 1, pp. 2-13, 2010.

[123] Y. Zhang, M. Adachi, R. Kawamura et al., "Bmf contributes to histone deacetylase inhibitor-mediated enhancing effects on apoptosis after ionizing radiation," Apoptosis, vol. 11, no. 8, pp. 1349-1357, 2006.

[124] K. Camphausen, D. Cerna, T. Scott et al., "Enhancement of in vitro and in vivo tumor cell radiosensitivity by valproic acid," International Journal of Cancer, vol. 114, no. 3, pp. 380-386, 2005.

[125] F. Zhang, T. Zhang, Z.-H. Teng, R. Zhang, J.-B. Wang, and Q.-B. Mei, "Sensitization to $\gamma$-irradiation-induced cell cycle arrest and apoptosis by the histone deacetylase inhibitor trichostatin A in non-small cell lung cancer (NSCLC) cells," Cancer Biology and Therapy, vol. 8, no. 9, pp. 823-831, 2009.

[126] K. S. Kumar, J. Sonnemann, L. T. T. Hong et al., "Histone deacetylase inhibitors, but not vincristine, cooperate with radiotherapy to induce cell death in medulloblastoma," Anticancer Research, vol. 27, no. 1 A, pp. 465-470, 2007.

[127] Y. Zhang, M. Adachi, X. Zhao, R. Kawamura, and K. Imai, "Histone deacetylase inhibitors FK228, N-(2-aminophenyl)4-[N-(pyridin-3-yl-methoxycarbonyl)amino-methyl]benzamide and $\mathrm{m}$-carboxycinnamic acid bis-hydroxamide augment radiation-induced cell death in gastrointestinal adenocarcinoma cells," International Journal of Cancer, vol. 110, no. 2, pp. 301-308, 2004.

[128] C. A. Banuelos, J. P. Banáth, S. H. MacPhail, J. Zhao, T. Reitsema, and P. L. Olive, "Radiosensitization by the histone deacetylase inhibitor PCI-24781," Clinical Cancer Research, vol. 13, no. 22, pp. 6816-6826, 2007.

[129] A. Munshi, T. Tanaka, M. L. Hobbs, S. L. Tucker, V. M. Richon, and R. E. Meyn, "Vorinostat, a histone deacetylase inhibitor, enhances the response of human tumor cells to ionizing radiation through prolongation of $\gamma$-H2AX foci," Molecular Cancer Therapeutics, vol. 5, no. 8, pp. 1967-1974, 2006.

[130] K. Camphausen, T. Scott, M. Sproull, and P. J. Tofilon, "Enhancement of xenograft tumor radiosensitivity by the histone deacetylase inhibitor MS-275 and correlation with histone hyperacetylation," Clinical Cancer Research, vol. 10, no. 18 I, pp. 6066-6071, 2004.

[131] I. A. Kim, J. H. Shin, I. H. Kim et al., "Histone deacetylase inhibitor-mediated radiosensitization of human cancer cells: class differences and the potential influence of p53," Clinical Cancer Research, vol. 12, no. 3 I, pp. 940-949, 2006.

[132] M. S. Kim, M. Blake, J. H. Baek, G. Kohlhagen, Y. Pommier, and F. Carrier, "Inhibition of histone deacetylase increases cytotoxicity to anticancer drugs targeting DNA," Cancer Research, vol. 63, no. 21, pp. 7291-7300, 2003.

[133] R. Stupp, W. P. Mason, M. J. Van Den Bent et al., "Radiotherapy plus concomitant and adjuvant temozolomide for glioblastoma," New England Journal of Medicine, vol. 352, no. 10, pp. 987-996, 2005.

[134] M. Entin-Meer, X. Yang, S. R. VandenBerg et al., "In vivo efficacy of a novel histone deacetylase inhibitor in combination with radiation for the treatment of gliomas," Neuro-Oncology, vol. 9, no. 2, pp. 82-88, 2007.

[135] D. Siegel, M. Hussein, C. Belani et al., "Vorinostat in solid and hematologic malignancies," Journal of Hematology and Oncology, vol. 2, article no. 31, 2009.

[136] M. A. Dimopoulos, J. F. San-Miguel, and K. C. Anderson, "Emerging therapies for the treatment of relapsed or refractory multiple myeloma," European Journal of Haematology, vol. 86, no. 1, pp. 1-15, 2011.

[137] L. R. Silverman, A. Verma, R. Odchimar-Reissig et al., "A phase I trial of the epigenetic modulators vorinostat, in combination with azacitidine (azaC) in patients with the Myelodysplastic Syndrome (MDS) and Acute Myeloid Leukemia (AML): a study of the New York Cancer Consortium," ASH Annual Meeting Abstracts, vol. 112, no. 11, p. 3656, 2008.

[138] S. D. Gore, A. Jiemjit, L. B. Silverman et al., "Combined methyltransferase/histone deacetylase inhibition with 5azacitidine and ms-275 in patients with MDS, CMMoL and AML: clinical response, histone acetylation and DNA damage," ASH Annual Meeting Abstracts, vol. 108, no. 11, p. $517,2006$.

[139] R. W. Johnstone and J. D. Licht, "Histone deacetylase inhibitors in cancer therapy: is transcription the primary target?" Cancer Cell, vol. 4, no. 1, pp. 13-18, 2003. 

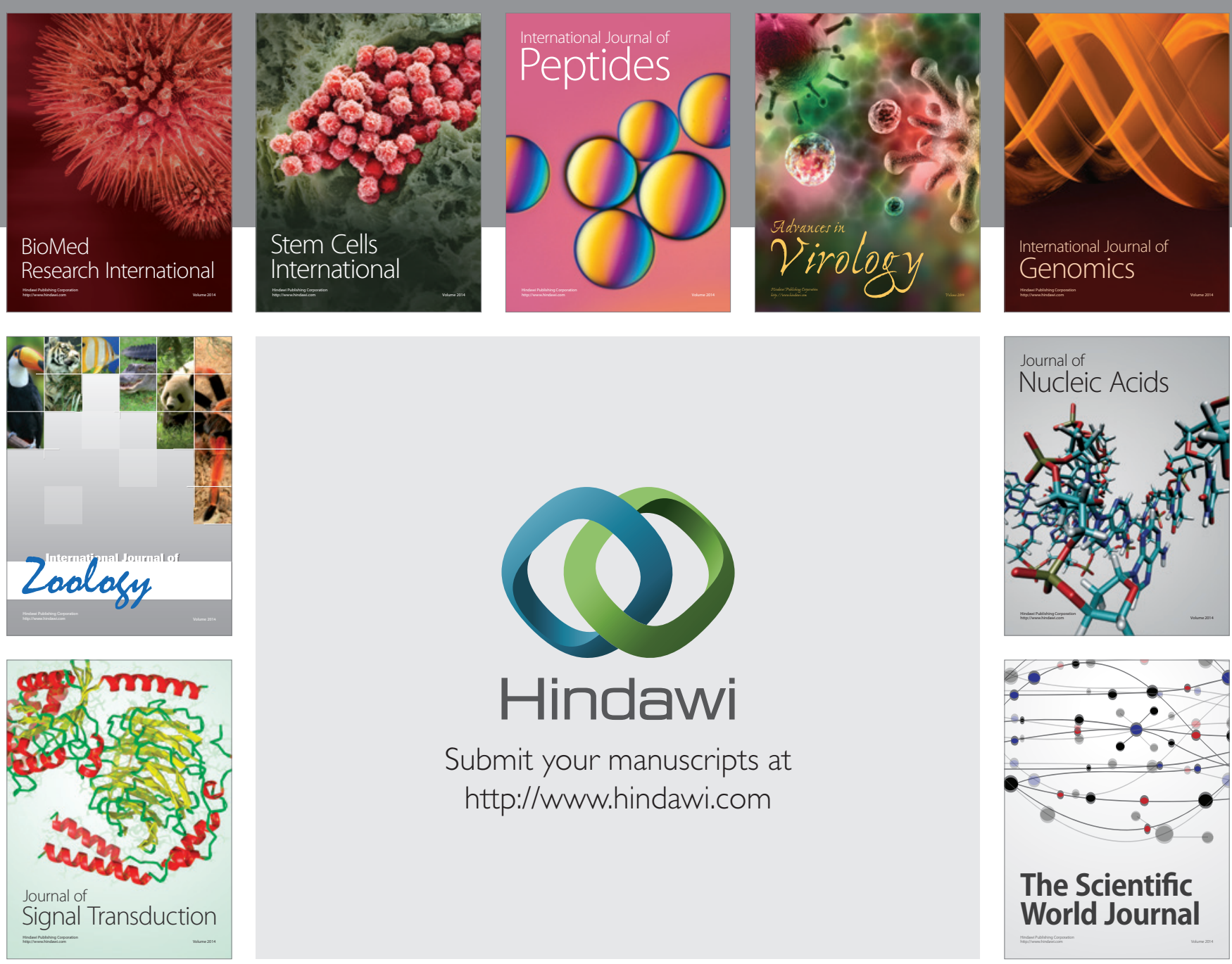

Submit your manuscripts at

http://www.hindawi.com
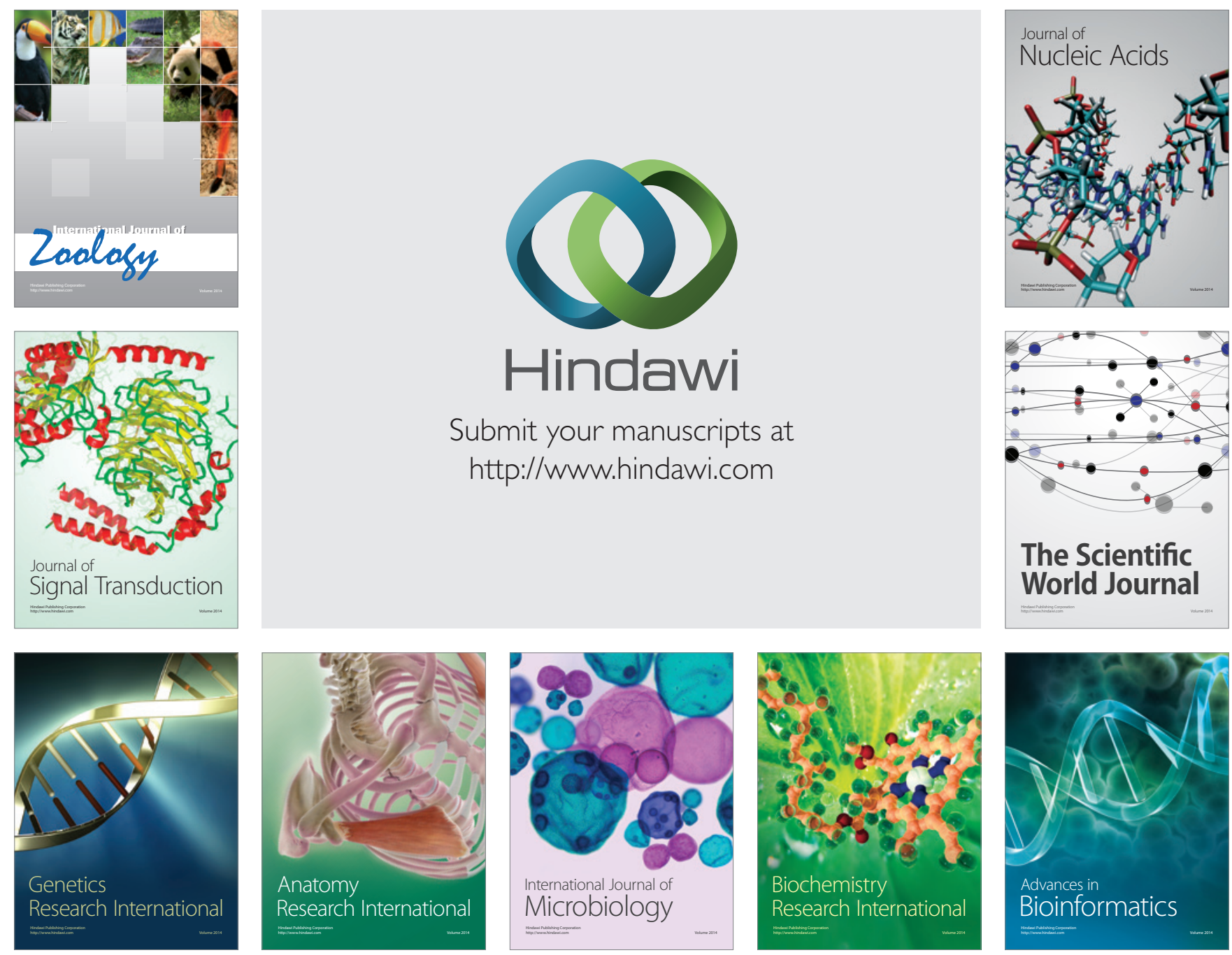

The Scientific World Journal
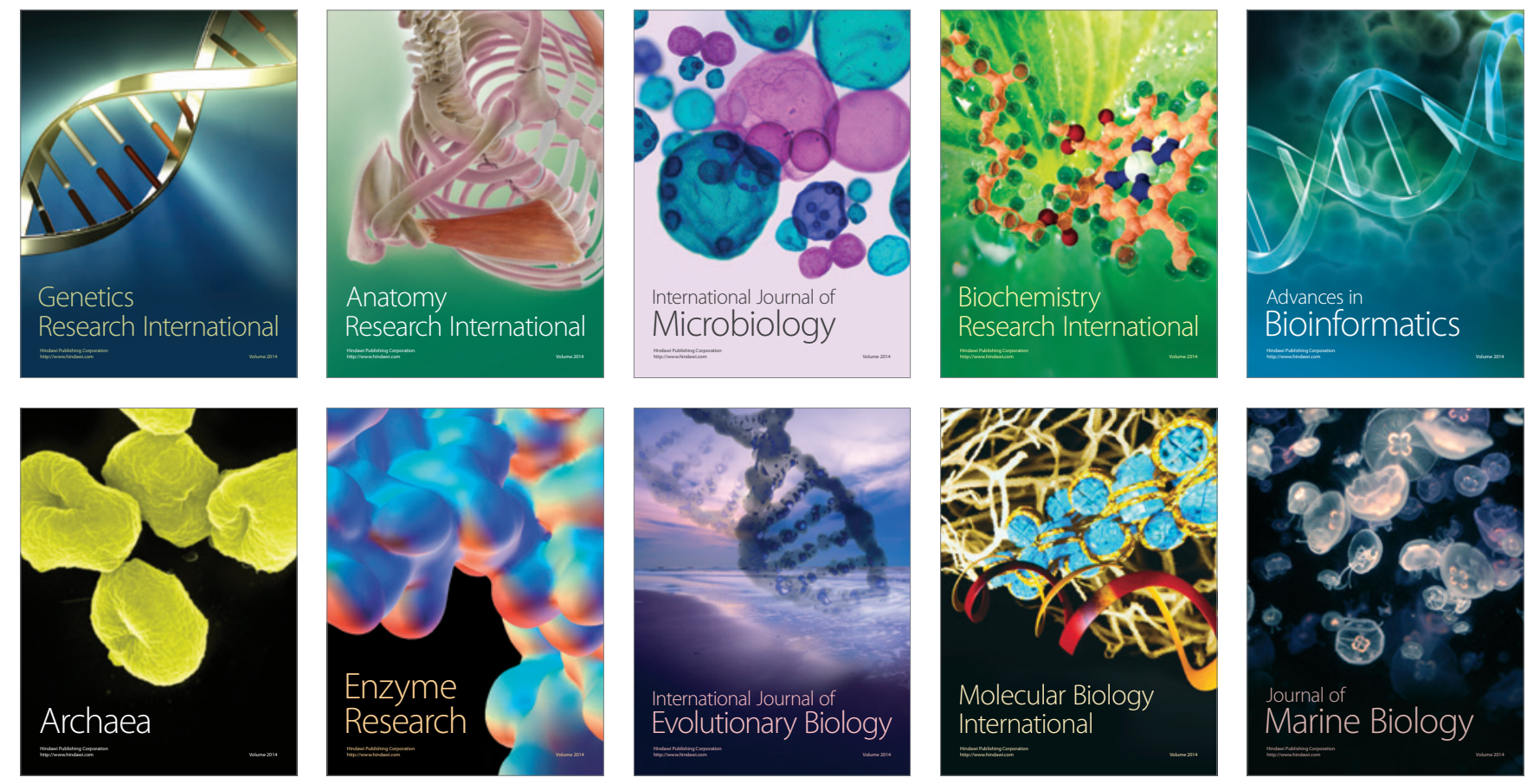